\title{
Hand-held Dual-particle Imager Implemented with a Multiplexed Low Sampling-rate Readout of a SiPM- based Pixelated Stilbene Array
}

Jihwan Boo

Jeju National University

Mark Hammig

University of Michigan-Ann Arbor

Manhee Jeong ( $\sim$ mhjeong@jejunu.ac.kr)

Jeju National University

\section{Research Article}

Keywords: particle imaging, gamma-rays, environment, dual particle imagers (DPIs), nalog-to-digital converters (ADCs)

Posted Date: December 9th, 2020

DOl: https://doi.org/10.21203/rs.3.rs-116991/v1

License: (9) This work is licensed under a Creative Commons Attribution 4.0 International License. Read Full License

Version of Record: A version of this preprint was published at Scientific Reports on February 15th, 2021. See the published version at https://doi.org/10.1038/s41598-021-83530-4. 


\title{
Hand-held dual-particle imager implemented with a multiplexed low sampling-rate readout of a SiPM-based pixelated stilbene array
}

\author{
Jihwan Boo ${ }^{1}$, Mark D. Hammig ${ }^{2}$, Manhee Jeong ${ }^{1 *}$ \\ ${ }^{1}$ Department of Nuclear and Energy Engineering, Jeju National University, Jeju 63243, \\ Republic of Korea \\ ${ }^{2}$ Department of Nuclear Engineering \& Rad. Sci., University of Michigan-Ann Arbor, MI- \\ 48109, USA \\ * Corresponding author: mhjeong@jejunu.ac.kr
}

\begin{abstract}
Dual particle imaging, in which both neutrons and gamma-rays in the environment can be individually characterized, is particularly attractive for monitoring mixed radiation emitters such as special nuclear materials (SNM). Typical dual particle imagers (DPIs) are not readily deployable and easily portable for hand-held applications because they are implemented using bulky single-crystal scintillators and photomultiplier tubes (PMTs) implemented with a 1:1 channel readout. Effective SNM localization and detection also benefits from high instrument sensitivity so that real-time imaging or imaging with a limited number of acquired events is enabled. We have developed a hand-held type DPI equipped with a pixelated stilbene-silicon photomultiplier (SiPM) array module and low sampling-rate analog-to-digital converters (ADCs) processed via a multiplexed readout. The stilbene-SiPM array $(12 \times 12$ pixels $)$ is capable of effectively performing pulse shape discrimination (PSD) between gamma-ray and neutron events and neutron/gamma-ray source localization on the imaging plane, as demonstrated with ${ }^{252} \mathrm{Cf}$ neutron/gamma and ${ }^{137} \mathrm{Cs}$ gamma-ray sources. The low sampling rate ADCs connected to the stilbene-SiPM array module result in a compact instrument with high sensitivity that provides a gamma-ray image of a ${ }^{137} \mathrm{Cs}$ source, producing $6.4 \mu \mathrm{R} / \mathrm{h}$ at $1 \mathrm{~m}$, in
\end{abstract}


less than 69 seconds. A neutron image for a $3.5 \times 10^{5} \mathrm{n} / \mathrm{s}{ }^{252} \mathrm{Cf}$ source can also be obtained in less than 6 minutes at $1 \mathrm{~m}$ from the center of the system. The instrument images successfully with field of view of $50^{\circ}$ and provides angular resolution of $6.8^{\circ}$.

\section{Introduction}

The nuclear safeguarding of special nuclear materials (SNM), such as plutonium and highly-enriched uranium, requires instruments that can detect, localize, and quantify the isotopic content of the target [1]. The accuracy of the material assessment can be enhanced if both the neutron and gamma-ray emissions are interrogated by a dual-particle imager (DPI). When coupled with a neutron-interrogating source, a DPI based on electronic collimation can localize a highly enriched uranium (HEU) solid surrounded by neutron shielding and distinguish it from a dummy material item such as tungsten [2]. The DPI system described in [3], which utilizes a coded aperture, successfully images both ${ }^{241} \mathrm{AmBe}$ and ${ }^{252} \mathrm{Cf}$ photon/neutron sources.

Despite these demonstrations, DPI systems have not yet been designed to be more portable and deployable or to maintain sufficiently high sensitivity to rapidly reconstruct images. The aforementioned DPI using electronic collimation employed a large, bulky single crystal array of EJ-309 and NaI:Tl coupled to photomultiplier tubes (PMTs). Furthermore, the implemented 1:1 channel readout was performed with cumbersome digitizers such as the CAEN V1720, which cannot be applied to hand-held use due to its size. A previous study on this DPI system also quantified the low efficiency $\left(10^{-4}\right.$ per incident neutron or photon) with which it created imageable events [4]. On the other hand, the DPI system in [3] based on the coded aperture has an array of bulky single crystals whose size are inversely proportional to the angular resolution of the system. In addition to its size, it took an hour each to measure the sources using mask and anti-mask measurement configurations, an approach that prevents real-time imaging. 
In [5], the authors implemented a pixelated detector in a hand-held DPI configuration using pulse-shape discrimination (PSD). The instrument, which consisted of a plastic scintillator (EJ299-34) $13 \times 13$ square array with a small pixel size $\left(2.8 \times 2.8 \times 15 \mathrm{~mm}^{3}\right)$, exhibited a marked degradation of PSD performance in the pixelated crystal array when compared to a single crystal. The PSD performance of the pixelated array, however, could not yet be fully verified because the authors detected the ${ }^{252} \mathrm{Cf}$ stored in a water tank that reduced the average energy $(2.1 \sim 2.5 \mathrm{MeV}$ ) of the fast neutron by one-third (to $0.7 \sim 0.9 \mathrm{MeV}$ ). Furthermore, because the estimation of PSD performance was performed across the entire energy spectrum, the results were biased toward the low energy events that generally represent the highest misclassification probability in most organic scintillators [6]. They were also not capable of showing pixel-bypixel PSD performance because the pixelated array was mounted on a single-channel photomultiplier tube (PMT) rather than pixelated photodetectors, such as silicon photomultiplier (SiPM).

There has been recent efforts to develop a hand-held DPI system [7] using high sampling rate analog-to-digital converters (ADCs), in which stilbene scintillator bars were coupled to an SiPM. Stilbene was chosen because it has high light output and high PSD performance when compared to other organic scintillators [8]. The SiPM also provides a small footprint and low voltage/power operation compared with PMTs. Nevertheless, the hand-held DPI system required high sampling rate ADCs (e.g., ADCs with 14 bits and $500 \mathrm{MS} / \mathrm{s}$ ) in order to provide sufficient time resolution to identify coincident interactions for time-of-flight (TOF) implementations. High-performance ADCs pose a limit in the use of portable DPI systems due to their high power consumption and large footprint. Because each stilbene bar is combined with two SiPM arrays and the 1:1 channel readout is implemented [9], increasing the number of these bars in future studies will double the number of readout channels and therefore increase the number of ADCs. An additional cooling system may be required for the ADCs unless a 
multiplexing method is implemented.

Another important performance consideration for many deployed or mobile applications is ensuring that the sensitivity of the DPI system is high enough to rapidly reconstruct images. As the SNM sample-to-detector distance increases, the validity of the SNM detection can rapidly decrease [10]. The aforementioned hand-held DPI system requires a long measurement time to reconstruct a neutron image of SNM as described in [7] due to its small number of stilbene bars. Furthermore, the complexity of the data collection system, such as determining the TOF between neutron interactions can increase dead-time losses and restrict the efficiency of the system. For instance, the minimum time difference between coincident neutron events should be 250 ps or more and the energy of the neutron after the initial scatter $\left(\mathrm{E}_{\mathrm{TOF}}\right)$ should be greater than the energy deposition in the second interaction. Thus, the minimum energy neutron required to generate an imageable event is roughly $1.5 \mathrm{MeV}$. The applied correction factor also created image artifacts in the reconstructed gamma-ray image because the entire energy of the gamma-ray is not fully deposited in the stilbene scintillators.

In this paper, we demonstrate the performance of a coded-aperture based hand-held DPI system using a pixelated stilbene-SiPM array module and low sampling rate ADCs. To our knowledge, this also represents the first experimental results of PSD performance when using a pixelated stilbene scintillator coupled to a SiPM array implemented with a multiplexing method such as row/column readout. The stilbene-SiPM array $(12 \times 12$ pixels $)$ module has high sensitivity that limits the measurement time for gamma-ray and neutron image acquisition. The coded-aperture based system utilizes the low sampling-rate ADCs to extract the relevant pulse information derived from neutron and gamma events in the detector module, while keeping the instrument mass of $4.1 \mathrm{~kg}$ and the device size compact.

\section{Results and discussion}


Figure 1 shows that all 144 elements of a stilbene-SiPM array module can be identified when exposed to either ${ }^{60} \mathrm{Co},{ }^{137} \mathrm{Cs},{ }^{22} \mathrm{Na},{ }^{133} \mathrm{Ba}$, or ${ }^{57} \mathrm{Co}$ gamma-ray sources $(0.312 \mathrm{MBq}$ activity) located $10 \mathrm{~cm}$ from the face of the module for an hour. As the gamma-ray energy decreases, the detection efficiency increases as expected; however, the positional resolution across the array is degraded owing to a higher relative participation of multi-pixel scattering events due to more intense sampling of large Compton scattering-angle events. Moreover, if there is optical cross-talk between pixels due to the imperfect capture of scintillation photons from the interaction pixel, then positional blurring and a mislocation of the interaction location can result. These negative impacts could be alleviated when considering only those events that occur close to the center of the pixel. The multiplexing scheme used here could set the acceptance range specified by the user as only those events that deposit their energy within $20 \%$ of the distance between the two pixels. This could be realized by the fact that the end of each row/column readout provided the signals that identified all interaction positions in real-time. This event processing manner could also give a positive effect on neutron measurement as we can effectively minimize multi-pixel events.

As shown in Fig. 2, energy spectra were acquired using the gamma-ray sources, and an energy calibration to units of keVee was made that had high accuracy $\left(>R^{2}=0.9999\right)$ in the range of Compton edge energy depositions between $40.385 \mathrm{keV}$ and $1061.71 \mathrm{keV}$. We confirmed that energy linearity was maintained after the electronic gain adjustment as expected because although there is pixel-by-pixel light yield and gain factor variation, those differences are compensated for by pixel-by-pixel gain calibrations for every element coupled to the SiPM [11]. An energy calibration to units of keVee was subsequently made using the linear light output response functions to photon interaction.

The pulse shape discrimination metric plot is shown in Fig. 3, which was acquired by measuring a $3.5 \times 10^{5} \mathrm{n} / \mathrm{s}{ }^{252} \mathrm{Cf}$ spontaneous fission source $50 \mathrm{~cm}$ distant from the detector 
module. The description and evaluation of the PSD metric is detailed in the Methods. Gammaray events are dominant below the dashed line shown in the figure while the neutron events are predominant above the dashed line. When classifying events based on the dashed line, one can acquire energy spectra of the gamma-ray or neutron detector events, as shown in Fig. 3. At the energy of $200 \mathrm{keVee}$, the overall detector PSD plot has a partial overlap between the gamma-ray and neutron distributions that makes it difficult to isolate the relevant particle species; however, once PSD sorting is applied for each pixel, as shown in Fig. 4, a clearer distinction between the neutron and gamma-ray events above the energy of $100 \mathrm{keVee}$ is observed. The measurements thus indicate that gamma/neutron discrimination is achievable on a pixel-by-pixel basis.

In addition to the PSD plot, a figure of merit (FOM) that measures the effectiveness of the gamma/neutron discrimination is defined in the Methods and shown graphically in Fig. 5b. The FOM profiles that visualize the neutron/gamma-ray separation at different energy regions: $300 \pm 100 \mathrm{keVee}, 500 \pm 100 \mathrm{keVee}, 700 \pm 100 \mathrm{keVee}$, are presented in Fig. 5, when the stilbene detector is exposed to combined ${ }^{252} \mathrm{Cf}$ and ${ }^{137} \mathrm{Cs}$ sources. The neutron/gamma ${ }^{252} \mathrm{Cf}$ source was located $75 \mathrm{~cm}$ distant and the ${ }^{137} \mathrm{Cs}$ gamma-ray source producing $30.16 \mu \mathrm{R} / \mathrm{h}$ was located $30 \mathrm{~cm}$ away from the detector module. The high intensity of counts in the 300 to $500 \mathrm{keVee}$ region of the gamma-ray section of the PSD plot correspond to the Compton edge and the Compton continuum regions of ${ }^{137} \mathrm{Cs}$ in Fig. 5a. This region buttresses the reliability of the PSD method and its relationship with energy correction to units of keVee. Figure 5c shows the PSD plot that one of the pixels has, and the corresponding FOM values higher than 1.55 were achieved for the aforementioned three cases, as shown in Fig. 5d. It should be noted that a single stilbene scintillator block coupled to the PMT shows better neutron/gamma separation performance when using digitizers with a sampling frequency of $500 \mathrm{MHz}$ and 14-bit resolution $[12,13]$. The values of the FOM quoted in these studies $(1.94 \sim 2.5)$ are 
considerably higher than those shown in the current study. This is because the stilbene scintillator pixels used here are not only smaller than those used in the reference studies, but also the pixels of SiPM used here have smaller active areas than the PMT. Nevertheless, because intrinsic particle separation of the scintillator is achieved [5], the low sampling rate ADCs were capable of performing satisfactory PSD using the pixelated stilbene-SiPM array module.

Sensitivity tests were conducted to study the reconstructed image quality and localization accuracy by increasing either the measurement time or the number of recorded events, as shown in Fig. 6. The ${ }^{252} \mathrm{Cf}$ spontaneous fission source was measured at $100 \mathrm{~cm}$ from the center of the system, using the hand-held DPI system equipped with a centered-mosaic modifieduniform redundant array (MURA) mask detailed in the Methods. The collected data were binned in an energy window [14] with a minimum threshold of 50 keVee. For quantitative and qualitative evaluation on the images, the reconstructed images were compared with reference ground-truth images (i.e. spatial distributions filled with 0 except for a 1 at the image center) through peak signal-to-noise ratio (PSNR) [15], normalized mean-square error (NMSE) [16], and structural similarity (SSIM) metrics [17]. If the PSNR value exceeds $33 \mathrm{~dB}$ or the NMSE value is close to 0 , or the SSIM value is close to 1 , it is judged that the reconstructed image is hard to distinguish from the original object.

Figures 6a-c show detector maps consisting of 330, 1,000, and 2,000 neutron events, respectively, classified as higher than the PSD value of 0.16 following the binning of the data. The corresponding MLEM images were reconstructed using each of these detector maps, as shown in Figs. 6d-f. These three images correspond to measurement of 1 minute, 3 minutes, and 6 minutes, respectively (a figure verified using repeated experiments). Table 1 presents each results of the neutron image evaluation using the three imaging metrics (PSNR, NMSE, SSIM) mentioned above. As the number of neutron interactions increased from 1,000 to 2,000, 
the PSNR metric surpassed $33 \mathrm{~dB}$ - and in fact, increase to $39.29 \mathrm{~dB}$, the NMSE decreased by more than an order of magnitude to $\sim 10^{-4}$, and SSIM approached closely to 1 , the combined evaluation of which indicated that effective localization was achieved and imaging artifacts were minimized. The experiments verified that the coded aperture-based DPI can provide effective neutron-source imaging with a limited number of counts and therefore rapid imaging for relatively intense or proximate neutron sources. This can be compared with H2DPI from the University of Michigan [5] that has a quoted localization time of 30 minutes for the neutron image of a $1.2 \times 10^{7} \mathrm{n} / \mathrm{s}^{252} \mathrm{Cf}$ source at $58.4 \mathrm{~cm}$ from the center of system.

Similar to neutron image evaluation, we estimated the quality of gamma-ray images by measuring the ${ }^{137} \mathrm{Cs}$ producing $6.4 \mu \mathrm{R} / \mathrm{h}$ at $100 \mathrm{~cm}$ from the hand-held DPI. Following the same process of binning the data, Fig. 7 presents each detector map consisting of 300, 600, and 1,000 gamma-ray events which are lower than the PSD value 0.16 , and MLEM images can be obtained using these detector maps, respectively. The three images in Fig. 7 correspond to measurement times of $20 \mathrm{~s}, 40 \mathrm{~s}$, and $69 \mathrm{~s}$, respectively, figure qualities verified via repeated measurements. The results of gamma-ray image evaluation using the three factors are presented in Table 2. As the classified count number surpasses 1,000, the high PSNR (50.65 $\mathrm{dB})$, low NMSE $\left(\sim 10^{-5}\right)$ and near-unity SSIM buttresses the qualitative evaluation of the Fig. 7 images which indicate that the gamma-ray source is effectively localized with minimal imaging artifacts. A previous study [18] in our laboratory supports this image-quality result because the number of approximately 1,000 counts per second provided real-time image reconstruction when using the hand-held coded aperture gamma-ray imaging system with a pixelated $12 \times 12$ inorganic scintillator-SiPM array module.

Figure 8 shows the test results that evaluate the maximum angular field of view (FOV) of the hand-held coded aperture based DPI system. The ${ }^{137} \mathrm{Cs}$ gamma-ray source position was varied over angles from $-25^{\circ}$ to $+25^{\circ}$, located $100 \mathrm{~cm}$ away from the imaging device. The 
source position is well-reconstructed across the angular range. Note that the elongation in the $+25^{\circ}$ image compared to the $-25^{\circ}$ image is due to the count sharing between neighboring pixels on the coarsely sampled image plane. Moreover, compared with the $\sim 1,000$ counts required to form high-quality images for sources near the center of the FOV, an average number of 1,800 events was required to obtain acceptable accuracy of the source position and image quality. The increased measurement time was needed to account for the increased participation of gamma-rays that were scattered by the mask itself at the higher incident angles. This degradation mechanism can be mitigated by using energy windowing techniques that sort out only the Compton edge events of ${ }^{137} \mathrm{Cs}[19]$.

For the angular resolution demonstration, we previously established that the angular resolution derived from a GAGG:Ce gamma-ray imaging system was $6.8^{\circ}$ [18]. The $3.5 \times 10^{5}$ $\mathrm{n} / \mathrm{s}{ }^{252} \mathrm{Cf}$ spontaneous fission source at $75 \mathrm{~cm}$ distance and a ${ }^{137} \mathrm{Cs}$ source producing $2.71 \mu \mathrm{R} / \mathrm{h}$ at $30 \mathrm{~cm}$ were thus separated by an angle of $6.8^{\circ}$. When utilizing the detector map obtained from gamma-ray events that are in the energy range above $50 \mathrm{keVee}$ and have the PSD value less than 0.16 , the two sources were distinguished in the gamma-ray image using MLEM as shown in Figure 9a. It should be noted that on average 7,000 gamma-ray events (2-minute measurement time), through repetitive experiments, ensured the successful separation of two sources in the image. The neutron image in Figure $9 \mathrm{~b}$ shows a hot-spot in the correct ${ }^{252} \mathrm{Cf}$ position and no source in the ${ }^{137} \mathrm{Cs}$ location. An average of 2,000 neutron events (4-minute measurement time), are required to retain the image quality and localization accuracy. A higher overall count was required to obtain these source images because the signal-to-noise ratio decreases as multiple sources are exposed to the coded aperture imager [20].

Figure 10 shows the radiographic images superimposed upon an optical image generated by a complementary metal oxide-semiconductor (CMOS) image sensor (IMX214 produced by SONY). In image superimposition processing, homography estimation [21] was used to 
convert a distorted plane viewed from the front into a two-dimensional image plane that has a perspective projection. Similar to performing a perspective transformation, matching between two images can be performed through the coordinate shift information of nine corresponding points, and the homography matrix can be obtained from matching information using the random sample consensus (RANSAC) algorithm [22]. When using the calculated homography matrix we were able to reconstruct the scene and infer the presence and location of mixed gamma-ray and neutron point sources.

\section{Conclusions}

In this paper, a hand-held coded aperture based DPI system was realized by processing the signals from a $12 \times 12$ pixelated stilbene-SiPM array module via a row/column readout. The coded aperture based system maintained a weight of $4.1 \mathrm{~kg}$ and compact size by utilizing low sampling-rate $\mathrm{ADCs}(50 \mathrm{MHz})$ that extract the relevant pulse information derived from neutron and gamma-ray events in the detector module. The system shows excellent energy linearity, good neutron/gamma discrimination ability, intrinsic detection efficiency, and imaging quality when measuring various gamma-ray sources and a ${ }^{252} \mathrm{Cf}$ spontaneous fission source. The low sampling rate ADCs connected to the stilbene-SiPM array module are capable of performing satisfactory PSD from the stilbene scintillator. In addition, future plans include the upgrade of the ADCs that have 14-bit resolution to improve PSD performance [23] while retaining the present sampling rate of $50 \mathrm{MHz}$. We will also study to minimize optical cross-talk between pixels and multi-pixel events by considering only those events that occur close to the center of the pixel because the row/column readout identified all interaction positions in real-time.

\section{Methods}

\section{Hardware fabrication and configuration}


This hand-held DPI system is developed for neutron/gamma detection by using the $12 \times$ 12 stilbene scintillator array (produced by Inrad Optics), which has a $50.02 \times 50.02 \mathrm{~mm}^{2}$ active area and $4.2 \mathrm{~mm}$ pitch, and a SiPM array sensor (ArrayC-30035-144P) with the same number and area of pixels, as shown in Fig. 11. The readout scheme, implemented with the circuit shown schematically in Fig. 12 and realized in Fig. 13, makes use of a row/column readout with a resistive divider [24]. The readout circuit reduces the standard outputs from 144 to 12 for each orthogonal $\mathrm{X}$ and $\mathrm{Y}$ direction in which the interaction position of individual scintillation events is determined. The $\mathrm{X}$ and $\mathrm{Y}$ line outputs are connected to 24 transimpedance preamplifiers whose gain is $100 \mathrm{Ohms}$, followed by individual shaping amplifiers. The energy of the event was calculated as the sum of all peak values in the rows or columns received within a predetermined (coincident) time window.

The amplified analog signals were digitized by three low-sampling rate ADCs $(50 \mathrm{MHz}, 8$ channel, 12-bit, ADS5281, Texas Instruments). The field programmable gate arrays (FPGA) sample the digitized signals arriving at the ADC input and compares the discrete digital value with the trigger level set in the FPGA. If the new ADC value exceeds the trigger level, its value is stored in a buffer and transmitted to the internal computer that is used for running the MLEM algorithm from which the source image is reconstructed. The image data is then transferred via Wi-Fi or transmission control protocol/internet protocol (TCP/IP) to a remote side PC such as a desktop or laptop. The axil fan (UF-92B23 produced by Fulltech) is also equipped to cool down the heat emission of the ADCs and maintain the performance of SiPM independently of temperature. The power drive unit uses an adapter power of $5 \mathrm{~V} \mathrm{DC} / 6 \mathrm{~A}$ as input power. It is designed to meet the current required for device driving and can be replaced by a battery in the near future.

As shown in Fig. 14a, we developed a $21 \times 21$ rank, centered-mosaic, MURA mask composed of a $2 \mathrm{~cm}$ thick tungsten [11]. The tungsten-based mask was also chosen not only to 
block gamma-rays but also to scatter fast neutrons [25]. The mask selected an anti-symmetric quadratic residue array of size $21 \times 21(2 p-1)$ with the prime number $(p)$ of 11 to match the 11

$\times 11$ pixel section of the SiPM. Previous work in our laboratory [3] has shown that this MURA mask reduces measurement time by half and successfully mitigates the artifacts without using the conventional anti-mask method. Figure $14 \mathrm{~b}$ shows the prototype hardware configuration equipped with the MURA mask. The total weight of the hardware, including the MURA mask and the tungsten shield (with a $1 \mathrm{~cm}$ thickness) enclosing the stilbene-SiPM array, is approximately $4.1 \mathrm{~kg}$.

\section{Image reconstruction method}

The image reconstruction method uses the MLEM algorithm as the iterative image reconstruction technique. The MLEM is based on the log-maximization of the Poisson-like probability function and described in the following equation [14]:

$$
\lambda_{j}^{\text {new }}=\frac{\lambda_{j}^{\text {old }}}{\sum_{i} A_{i j}} \sum_{i} A_{i j} \frac{y_{i}}{\sum_{k} A_{i k} \lambda_{k}+b}
$$

where y contains the measured mask projection, in which $\mathrm{y}_{\mathrm{i}}$ is the number of counts recorded by detector pixel $\mathrm{i}$. That is, a point source is present in the projection because the projection of the source through the mask is deposited on the detector. $A$ is the system matrix consisting of the estimated mask projections from various source positions, where $A_{i j}$ is the predicted response of detector pixel $\mathrm{i}$ when the source is located in source-plane pixel $\mathrm{j}$. If there is a mismatch between the measured and estimated projection $\left(\lambda_{\mathrm{k}}\right.$, the ratio of two sets of projection), modifications are made to improve the estimate, and a new iteration is performed

until $\mathrm{k}$, the maximum iteration counter, is reached. This process leads to the maximized probability $\left(\lambda_{\mathrm{j}}\right)$ when the source is located at a source plane pixel $\mathrm{j}$. The term $\mathrm{b}$ is a noise term that represents the probability derived from the background radiation. Therefore, this process 
can readily estimate the position of the radiation source with a small number of incident radiation enough to form the mask projection.

\section{Neutron/gamma separation using PSD}

The charge comparison (CC) method was used in the PSD performance test, where the difference in the amount of delayed light produced by proton and electron recoil is used to identify neutron and $\gamma$-ray events, respectively. The capsule type ${ }^{252} \mathrm{Cf}$ source which has a 3.145 $\mathrm{MBq}$ activity was used as a fast neutron and multi energy gamma-ray source. Because ${ }^{252} \mathrm{Cf}$ emits an average of 3.759 neutrons and 8.3 gamma-rays per spontaneous fission event with a branching ratio of $3.09 \%$ [26], the source emitted $3.5 \times 10^{5} \mathrm{n} / \mathrm{s}$ into $4 \pi$ and $7.8 \times 10^{5} \mathrm{\gamma} / \mathrm{s}$. The PSD value is given by the equation:

$$
\text { PSD }=\frac{Q_{\text {tail }}}{Q_{\text {peak }}}=\frac{\int_{\text {tail }_{\text {start }}}^{\text {tail }_{\text {end }}} Q d t}{Q_{\text {peak }}}
$$

where tail $1_{\text {start }}$ and tail $l_{\text {end }}$ are the beginning and end of the tail signal, respectively, used for the integration of charge generated by delayed light, as shown in Fig. 15a. Qpeak corresponds to the charge of the peak due to prompt light. The sampling rate of the ADS5281 ADCs was set to 50 $\mathrm{MHz}$, for which the sampling interval is $20 \mathrm{~ns}$. The beginning of the tail used for total charge integration was fixed at 10 samples following the maximum peak amplitude, and the end of tail was fixed at 40 samples from the pulse maximum. Figure $15 \mathrm{~b}$ quantitatively describes the index of PSD performance as the FOM defined by the ratio of the peak center difference to the sum of the total width at half the maximum (FWHM) of the two peaks.

\section{Device Characterization}

Each of the three circuit boards consists of two developed printed circuit board (PCB) boards 
(front-end and charge division circuits) and a commercially available CPU board. The CPU board is equipped with an advanced RISC machine (ARM) CPU based on the Linux OS that utilizes a Raspberry PI board. The board performs (1) the image processing using the MLEM reconstruction method and (2) superimposes the resulting radiological images upon a concurrent optical image. The image data is then transferred to a remote side PC such as a desktop or laptop. The amount of data that can be transferred is up to 10 Mbyte per second, which translates into a neutron/gamma-ray count rate of up to $1,000,000 / \mathrm{s}$ [27].

\section{References}

1. Poitrasson-Rivière, Alexis, et al. Angular-resolution and material-characterization measurements for a dual-particle imaging system with mixed-oxide fuel. Nucl. Instrum. Methods. Phys. Res. B. 797, 278-284, DOI: https://doi.org/10.1016/j.nima.2015.06.045 (2015).

2. Hamel, M. C. et al. Active neutron and gamma-ray imaging of highly enriched uranium for treaty verification. Sci. Rep. 7, 1-10, DOI: https://doi.org/10.1038/s41598-017-08253$\underline{\mathrm{x}}(2017)$.

3. Hausladen, P. A. et al. Fast-neutron coded-aperture imaging of special nuclear material configurations. Idaho Nat. Lab., Idaho Falls, ID, USA, Tech. Rep. INL/CON-12-26506: (2012).

4. Poitrasson-Rivière, A. et al. Dual-particle imaging system based on simultaneous detection of photon and neutron collision events. Nucl. Instrum. Methods. Phys. Res. B. 760, 40-45, DOI: https://doi.org/doi:10.1016/j.nima.2014.05.056 (2014).

5. Cieślak, M. J., Gamage, K. A., Glover, R., \& Taylor, C. J. Pulse shape discrimination performance of a pixelated plastic scintillator (EJ-299-34) for a coded-aperture based dual particle imaging system. J. Instrum. 14, P07017. DOI: https://doi.org/10.1088/1748- 
$\underline{0221 / 14 / 07 / \mathrm{P} 07017}$ (2019).

6. Knoll, G. Radiation Detection and Measurement. (John Wiley \& Sons, 2010). 581-582.

7. Steinberger, William M., et al. Imaging Special Nuclear Material using a Handheld Dual Particle Imager. Sci. Rep. 10, 1-11. DOI: https://doi.org/10.1038/s41598-020-58857-z (2020).

8. Cieslak, M. Development of scintillator based coded-aperture neutron imager for nuclear decommissioning. Ph.D. thesis, University of Lancaster (2019).

9. Giha, N. P., Ruch, M. L., Di Fulvio, A., Steinberger, W. M., \& Pozzi, S. A. Readout electronics of a handheld dual particle imager. In 2017 IEEE Nucl. Sci. Symp. Atlanta, Georgia. 1-3, DOI: https://doi.org/10.1109/NSSMIC.2017.8532622 (2017).

10. Slaughter, D. et al. Detection of special nuclear material in cargo containers using neutron interrogation. Report UCRL-ID-155315. Lawrence Livermore National Laboratory, Livermore, California, DOI: https://doi.org/10.2172/15005260 (2003).

11. Jeong, M., Van, B., Wells, B. T., D’Aries, L. J., \& Hammig, M. D. Scalable Gamma-ray Camera for Wide-Area Search Based on Silicon Photomultipliers Array. Rev. Sci. Instrum. 89033106 DOI: https://doi.org/10.1063/1.5016563 (2018).

12. Ruch, M. L., Flaska, M., \& Pozzi, S. A. Pulse shape discrimination performance of stilbene coupled to low-noise silicon photomultipliers. Nucl. Instrum. Methods. Phys. Res. B. 793, 1-5. DOI: https://doi.org/10.1016/j.nima.2015.04.053 (2015).

13. Kim, G. Digital $n-\gamma$ pulse shape discrimination in organic scintillators with a high-speed digitizer. J. Radiat. Prot. Res. 44, 53-63. DOI: https://doi.org/10.14407/jrpr.2019.44.2.53 (2019).

14. Jeong, M., \& Hammig, M. D. Comparison of gamma ray localization using system matrixes obtained by either MCNP simulations or ray-driven calculations for a codedaperture imaging system. Nucl. Instrum. Methods. Phys. Res. B. 893, 161353. DOI: 
https://doi.org/10.1016/j.nima.2018.10.031 (2018).

15. Yan, Y., Cao, W., \& Li, S. Block-based adaptive image watermarking scheme using just noticeable difference, In IEEE I. W. Imag. Syst. Techni. 377-380. DOI: https://doi.org/10.1109/WICT.2011.6141267 (2009).

16. Chaves, R. et al., SVM-based computer-aided diagnosis of the Alzheimer's disease using t-test NMSE feature selection with feature correlation weighting. Neurosci. Lett. 461, 293297. DOI: https://doi.org/10.1016/j.neulet.2009.06.052 (2009).

17. Hore, A., \& Ziou, D. Image Quality Metrics: PSNR vs. SSIM. In 2010 20th international conference on pattern recognition. DOI: https://doi.org/10.1109/ICPR.2010.579 (2010).

18. Park, S., Boo, J., Hammig, M., \& Jeong, M. Impact of aperture-thickness on the real-time imaging characteristics of coded-aperture gamma cameras. Nucl. Eng. Technol. (in press). DOI: https://doi.org/10.1016/j.net.2020.09.012 (2020).

19. Liu, Q. et al., Image reconstruction using multi-energy system matrices with a scintillatorbased gamma camera for nuclear security applications. Appl. Radiat. Isot. 163, 109217. DOI: https://doi.org/10.1016/j.apradiso.2020.109217 (2020).

20. Brennan, J., et al. Demonstration of two-dimensional time-encoded imaging of fast neutrons. Nucl. Instrum. Methods. Phys. Res. B. 802, 76-81. DOI: https://doi.org/10.1016/j.nima.2015.08.076 (2015).

21. Dubrofsky, E., Homography estimation. Master's thesis, Carleton University. (2009).

22. Lee, J., \& Kim, G. Robust estimation of camera homography using fuzzy RANSAC. In: Gervasi, O., Gavrilova, Marina L. (eds.) ICCSA 2007. LNCS, 4705, 992-1002. Springer, Heidelberg. DOI: https://doi.org/10.1007/978-3-540-74472-6_81 (2007).

23. Flaska, M., Faisal, M., Wentzloff, D. D., \& Pozzi, S. A. Influence of sampling properties of fast-waveform digitizers on neutron- gamma-ray, pulse-shape discrimination for organic scintillation detectors. Nucl. Instrum. Methods. Phys. Res. B. 729, 456-462. DOI: 
https://doi.org/10.1016/j.nima.2013.07.008 (2013).

24. Popov, V., Majewski, S., \& Weisenberger, A. G. Readout Electronics for Multianode Photomultiplier Tubes With Pad Matrix Anode Layout. In 2003 IEEE Nucl. Sci. Symp. Conf. Rec. (IEEE Cat. No. 03CH37515). 3, 2156-2159. DOI: https://doi.org/10.1109/NSSMIC.2003.1352307 (2003).

25. Lister, D., Smith, A.B. and Dunford, C. Fast-neutron scattering from the 182, 184, and 186 isotopes of tungsten. Physical Review, 162, 1077. DOI: https://doi.org/10.1103/PhysRev.162.1077 (1967).

26. Al Hamrashdi, H., Cheneler, D., \& Monk, S. D. A fast and portable imager for neutron and gamma emitting radionuclides. Nucl. Instrum. Methods. Phys. Res. B. 953, 163253. DOI: https://doi.org/10.1016/i.nima.2019.163253 (2020).

27. Jeong, M., \& Hammig, M. Development of hand-held coded-aperture gamma ray imaging system based on GAGG (Ce) scintillator coupled with SiPM array. Nucl. Eng. Technol. (in press). DOI: https://doi.org/10.1016/j.net.2020.04.009 (2020).

\section{Acknowledgements}

This work was partly supported by Korea Institute of Energy Technology Evaluation and Planning (KETEP) grant funded by the Korea government (MOTIE) (20181520302230) and by the Nuclear Safety Research Program through the Korea Foundation of Nuclear Safety (KoFONS) using the financial resource granted by the Nuclear Safety and Security Commission (NSSC) of the Republic of Korea (No. 1903011-0119-CG100).

\section{Author Contributions}

M.D.H. and M.J. proposed the work and designed the experiments. M.J. fabricated and characterized the devices. J.B., M.D.H., and M.J. performed the analysis and discussed the 
result. All the authors contributed to the writing of the manuscript. 
Figure 1


${ }^{133}$ Ba Flood map
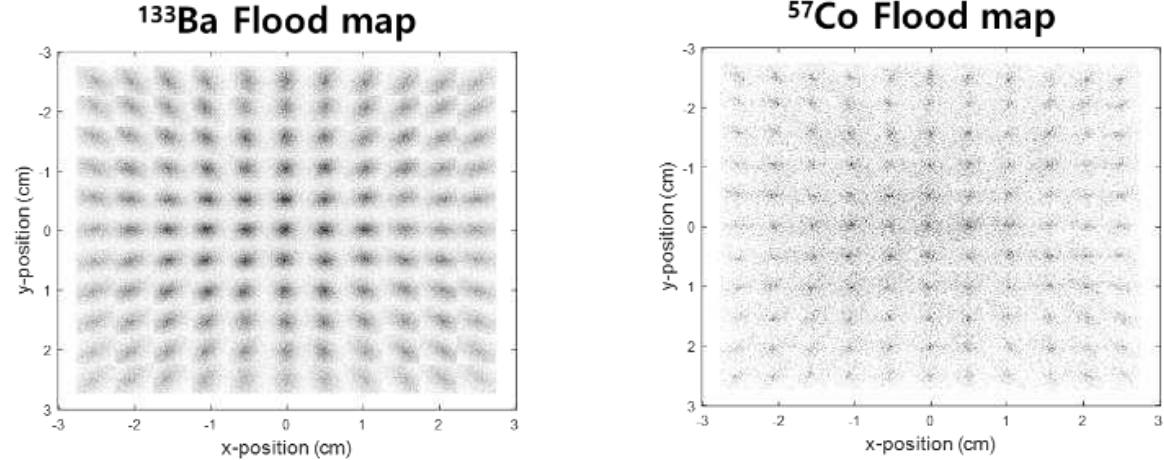

Figure 1. Measured 2D flood histograms of $12 \times 12$ pixels in stilbene-SiPM array module for ${ }^{60} \mathrm{Co},{ }^{137} \mathrm{Cs},{ }^{22} \mathrm{Na},{ }^{133} \mathrm{Ba}$, and ${ }^{57} \mathrm{Co}$ gamma irradiation. The source activity of each gamma-ray source was $0.312 \mathrm{MBq}$ and the $10 \mathrm{~cm}$ distant source was measured for 1 hour. The $12 \times 12$ stilbene scintillator had pixel dimensions $4 \times 4 \times 20 \mathrm{~mm}^{3}$. 
Figure 2

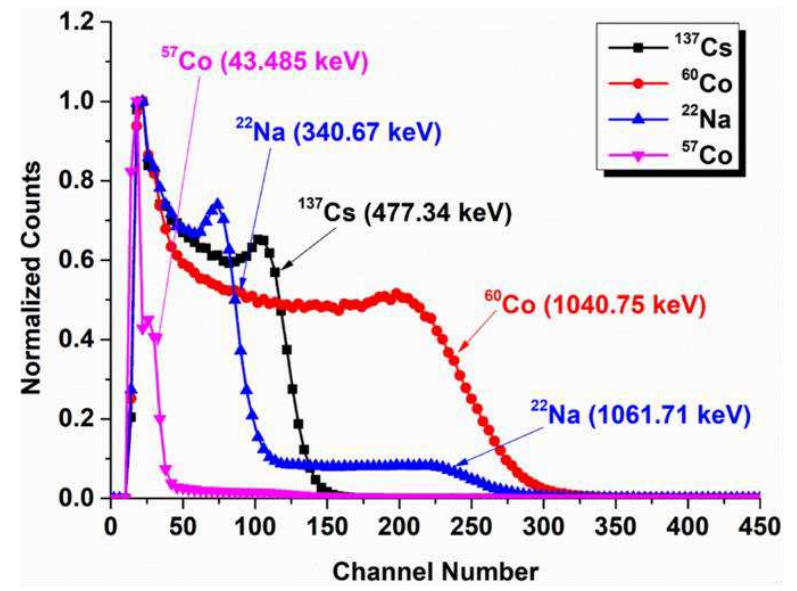

(a)

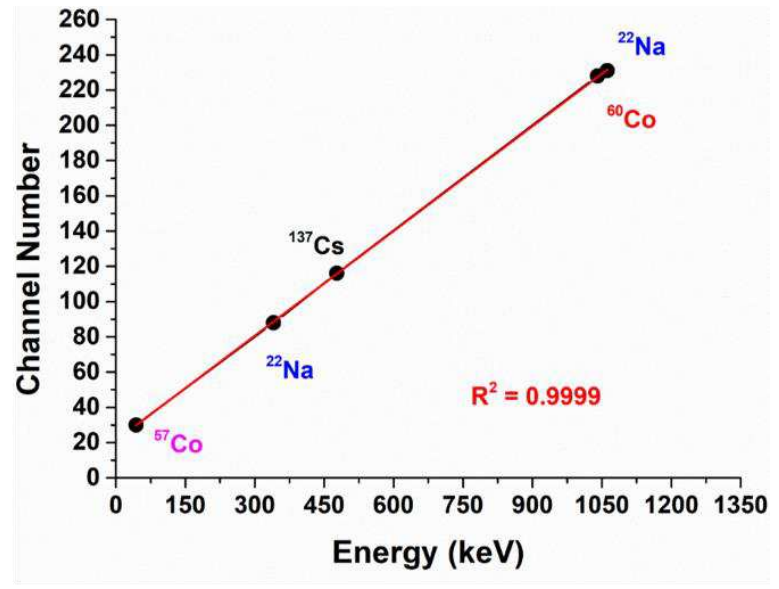

(b)

Figure 2. (a) Energy spectra of the stilbene scintillator array coupled to the SiPM array when irradiated by the gamma-ray sources shown. (b) Measured Compton edge height (in channel number) as a function of analytical Compton edge energy when the SiPM was operated at a 28 $\mathrm{V}$ bias voltage and a temperature of $28^{\circ} \mathrm{C}$. The Compton edge height was calculated at $70 \%$ of the Compton peak for each of the gamma-ray sources. 
Figure 3

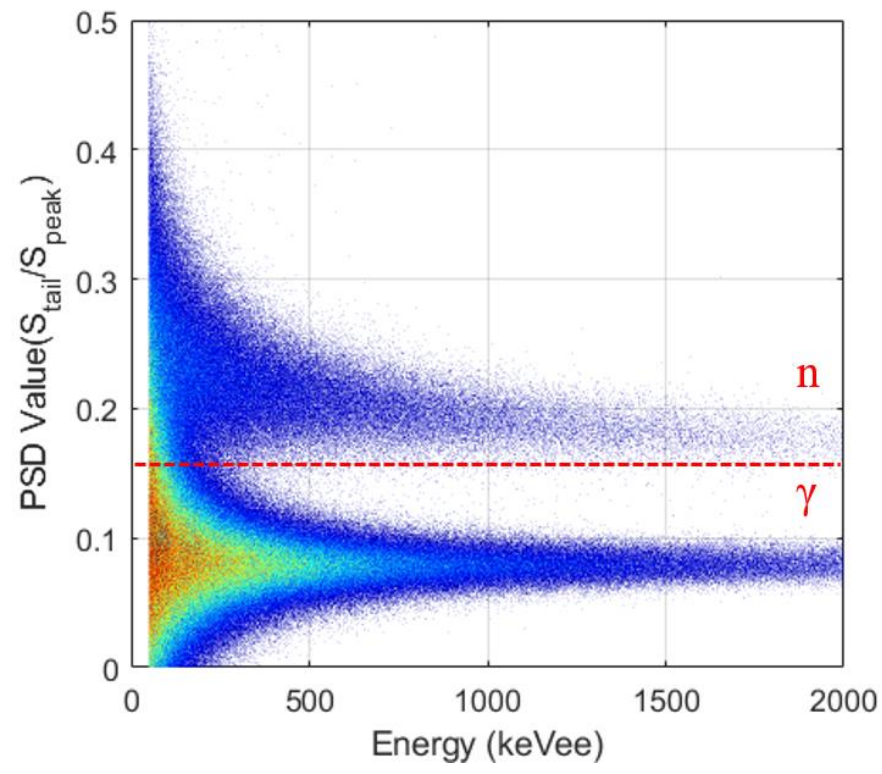

Figure 3. PSD plot for $1,800,000$ pulses with an energy threshold of $50 \mathrm{keVee}$ produced by the stilbene-SiPM array module measuring a $3.5 \times 10^{5} \mathrm{n} / \mathrm{s}^{252} \mathrm{Cf}$ spontaneous fission source located $50 \mathrm{~cm}$ away from the front of detector. The dashed PSD cutoff line is established by the user. 
Figure 4

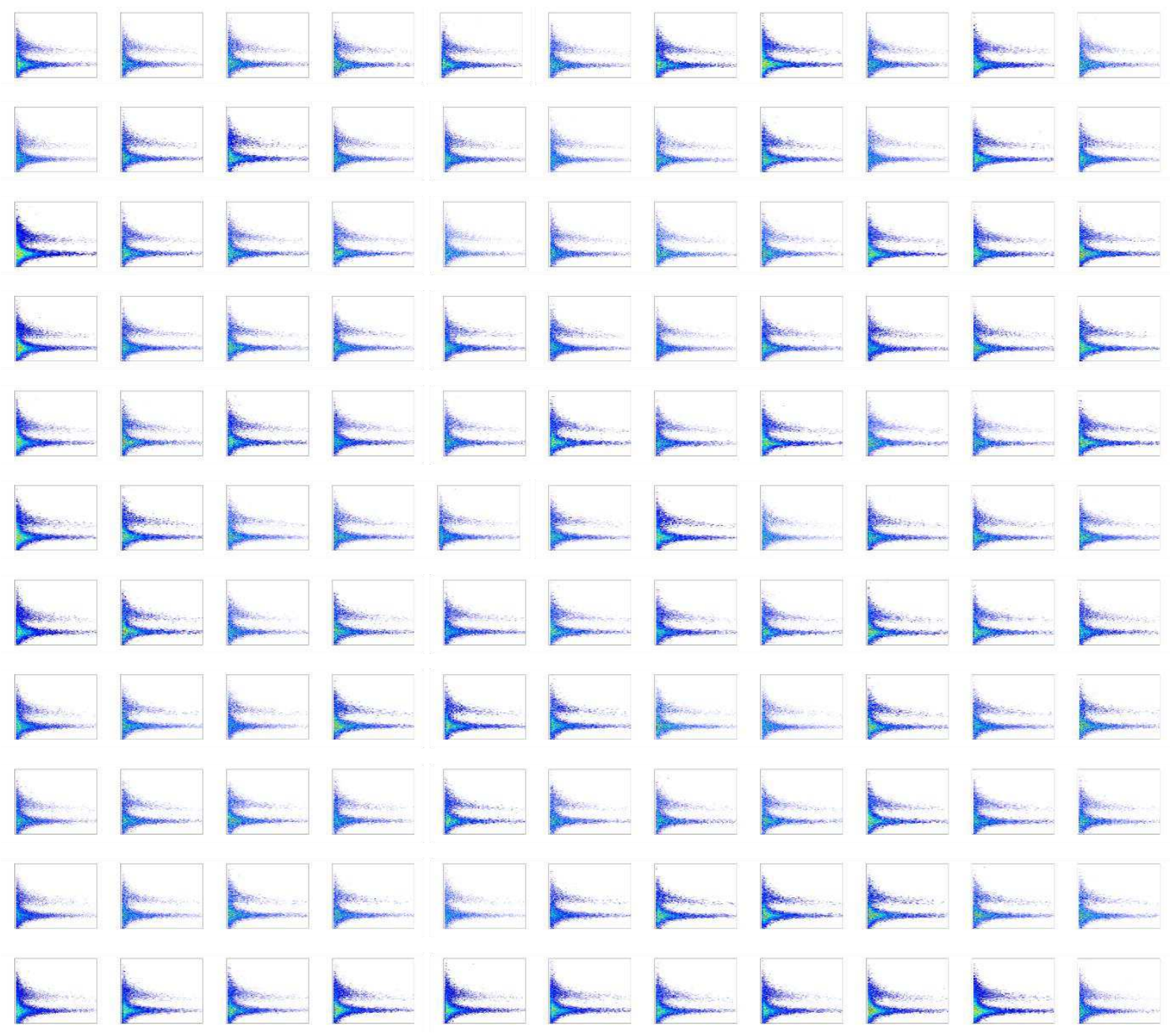

Figure 4. Pixel-by-pixel PSD plot for each $4 \times 4 \times 20 \mathrm{~mm}^{3}$ stilbene scintillator pixel from which the overall Fig. 3 distribution is generated. 
Figure 5


Figure 5. (a) The overall detector PSD plot for 1,500,000 pulses produced by the stilbene-SiPM module with a threshold of $50 \mathrm{keVee}$ by measuring a $3.5 \times 10^{5} \mathrm{n} / \mathrm{s}{ }^{252} \mathrm{Cf}$ spontaneous fission source at $75 \mathrm{~cm}$ distance and a ${ }^{137} \mathrm{Cs}$ source producing $30.16 \mu \mathrm{R} / \mathrm{h}$ at $30 \mathrm{~cm}$ distance. (b) The distribution of PSD parameter at various energies $(300 \pm 100 \mathrm{keVee}, 500 \pm 100 \mathrm{keVee}, 700 \pm 100$ keVee) for the overall detector PSD plot. (c) The PSD plot that one of the pixels has and (d) the corresponding FOM values for the aforementioned three cases. 
Figure 6

(a)

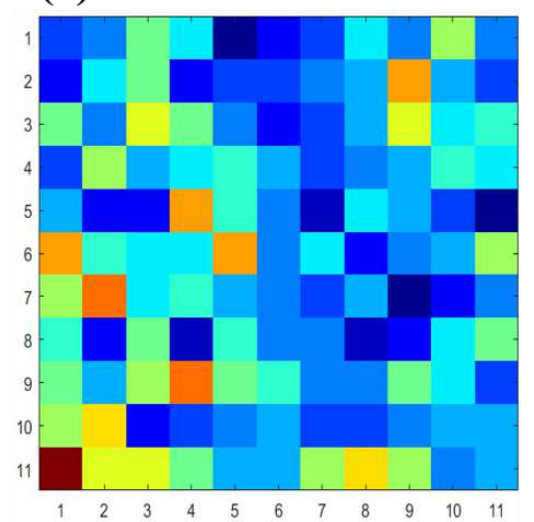

(d)

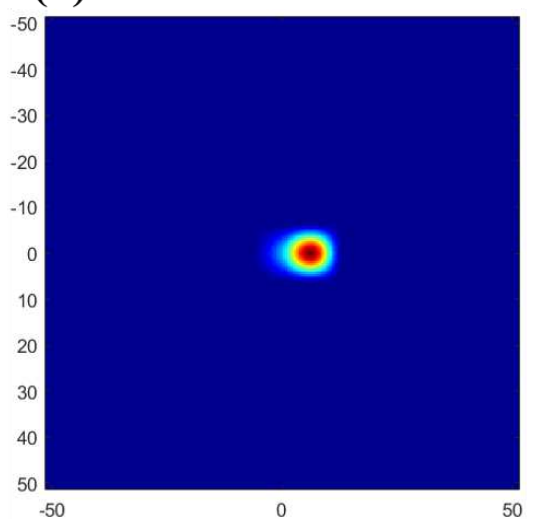

(b)

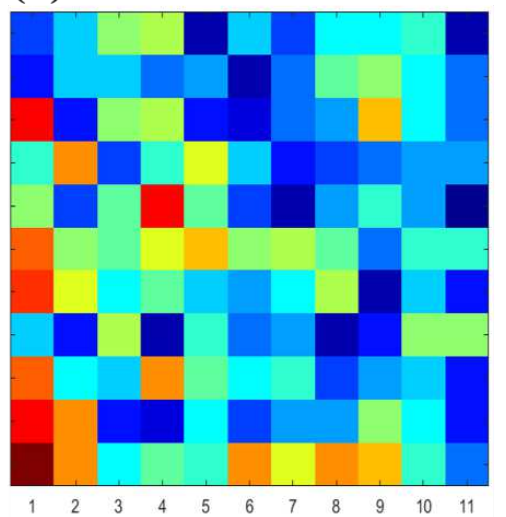

(e)

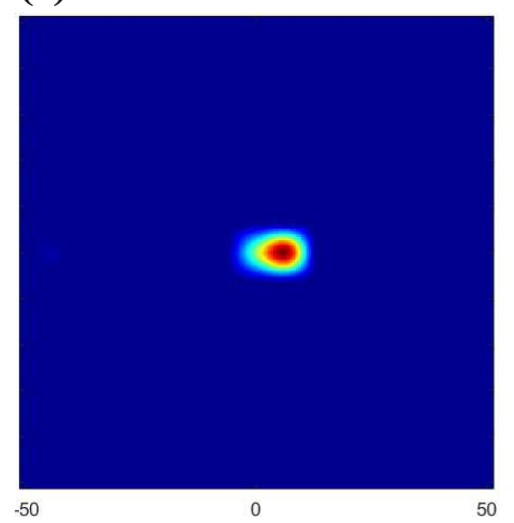

(c)

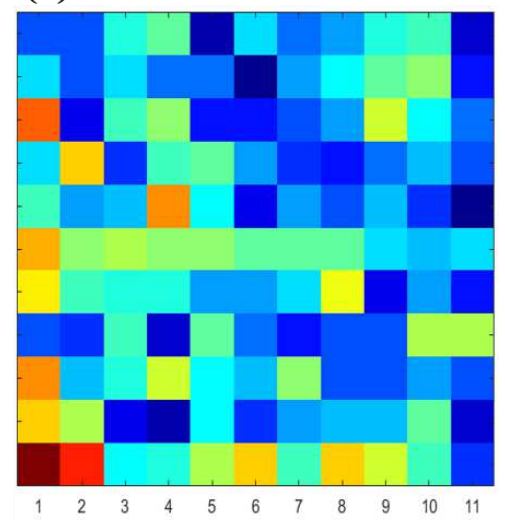

(f)

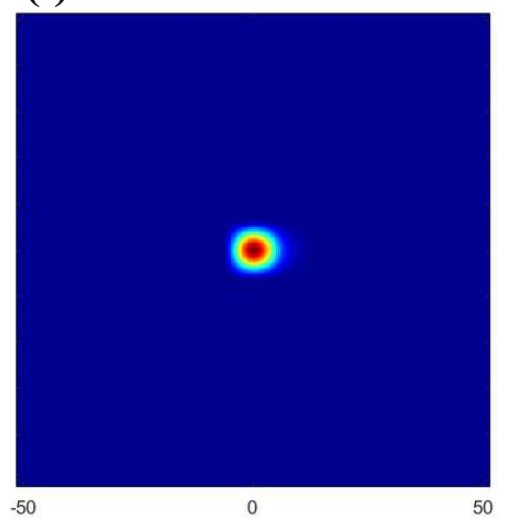

Figure 6. Comparison of reconstructed images of neutron when exposed to a $3.5 \times 10^{5} \mathrm{n} / \mathrm{s}{ }^{252} \mathrm{Cf}$ spontaneous fission source at $100 \mathrm{~cm}$ distance. Detector map composes of the neutron events obtained for 1 min (330 events) (a), 3 min (1,000 events) (b), and $6 \min (2,000$ events) (c). The three detector maps correspond to the images reconstructed by using MLEM (d-f), respectively.

Figure 7 
(a)

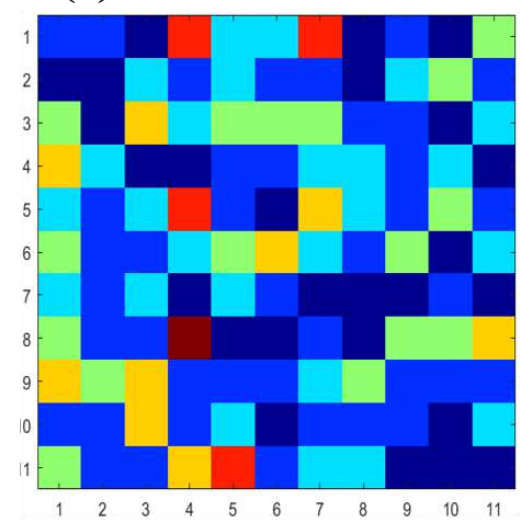

(d)

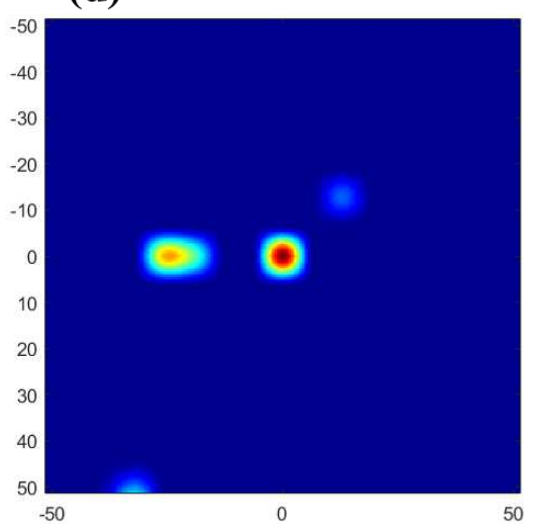

(b)

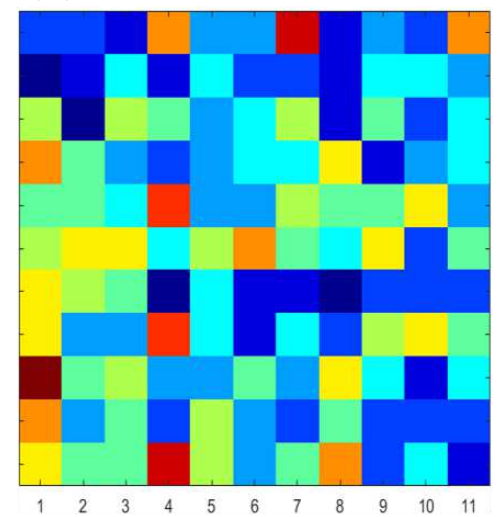

(e)

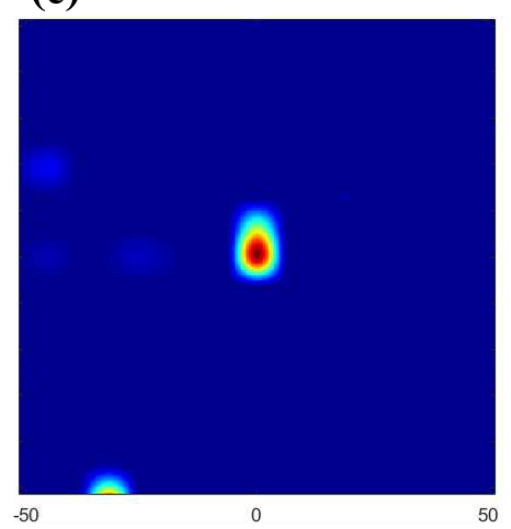

(c)



(f)

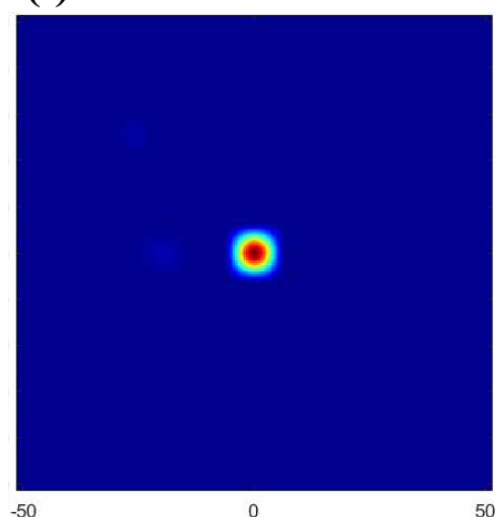

Figure 7. Comparison of reconstructed images of gamma-ray events when exposed to a ${ }^{137} \mathrm{Cs}$ source producing $6.4 \mu \mathrm{R} / \mathrm{h}$ at $100 \mathrm{~cm}$ distance. The detector map composed of the gamma-ray events for (a) $20 \mathrm{sec}$ (300 events), (b) $40 \mathrm{sec}$ (600 events), and (c) $69 \mathrm{sec}$ (1,000 events). The three detector maps correspond to the images reconstructed by using MLEM (d-f), respectively.

Figure 8 


\section{Field of View Test}

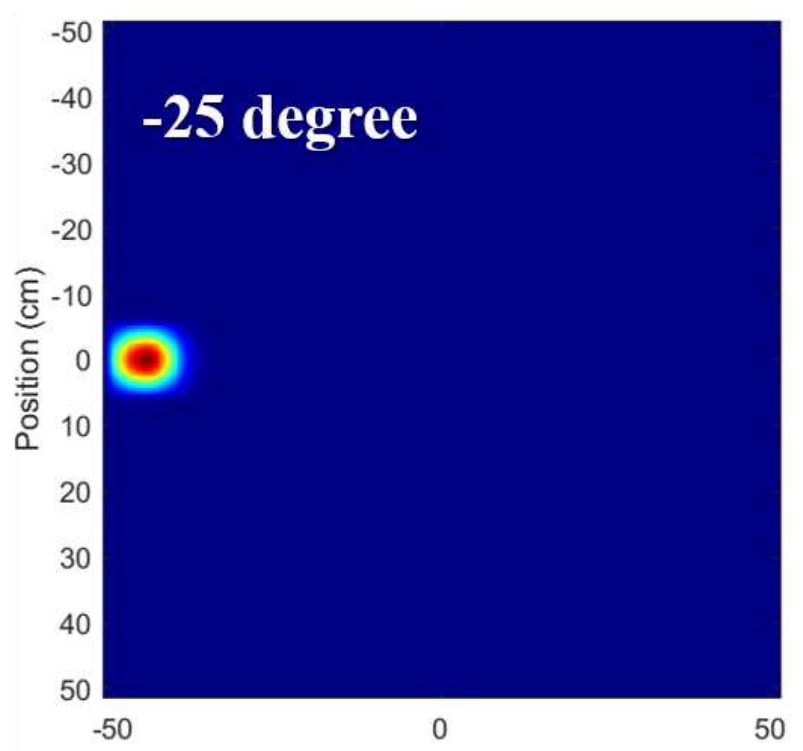

(a)

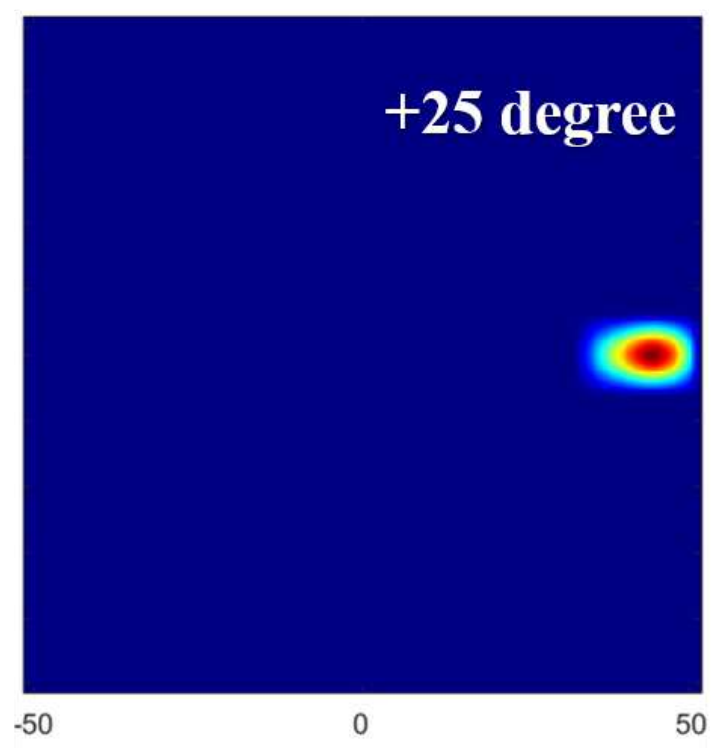

(b)

Position (cm)

Figure 8. Field of view (FOV) tests for the coded-aperture DPI system when a ${ }^{137} \mathrm{Cs}$ source of $0.312 \mathrm{MBq}$ located at an angle of $-25^{\circ}$ (a), and at $+25^{\circ}$ (b) at $100 \mathrm{~cm}$ distance. Both reconstructed images are obtained using the gamma-ray events above the 50 keVee. 
Figure 9

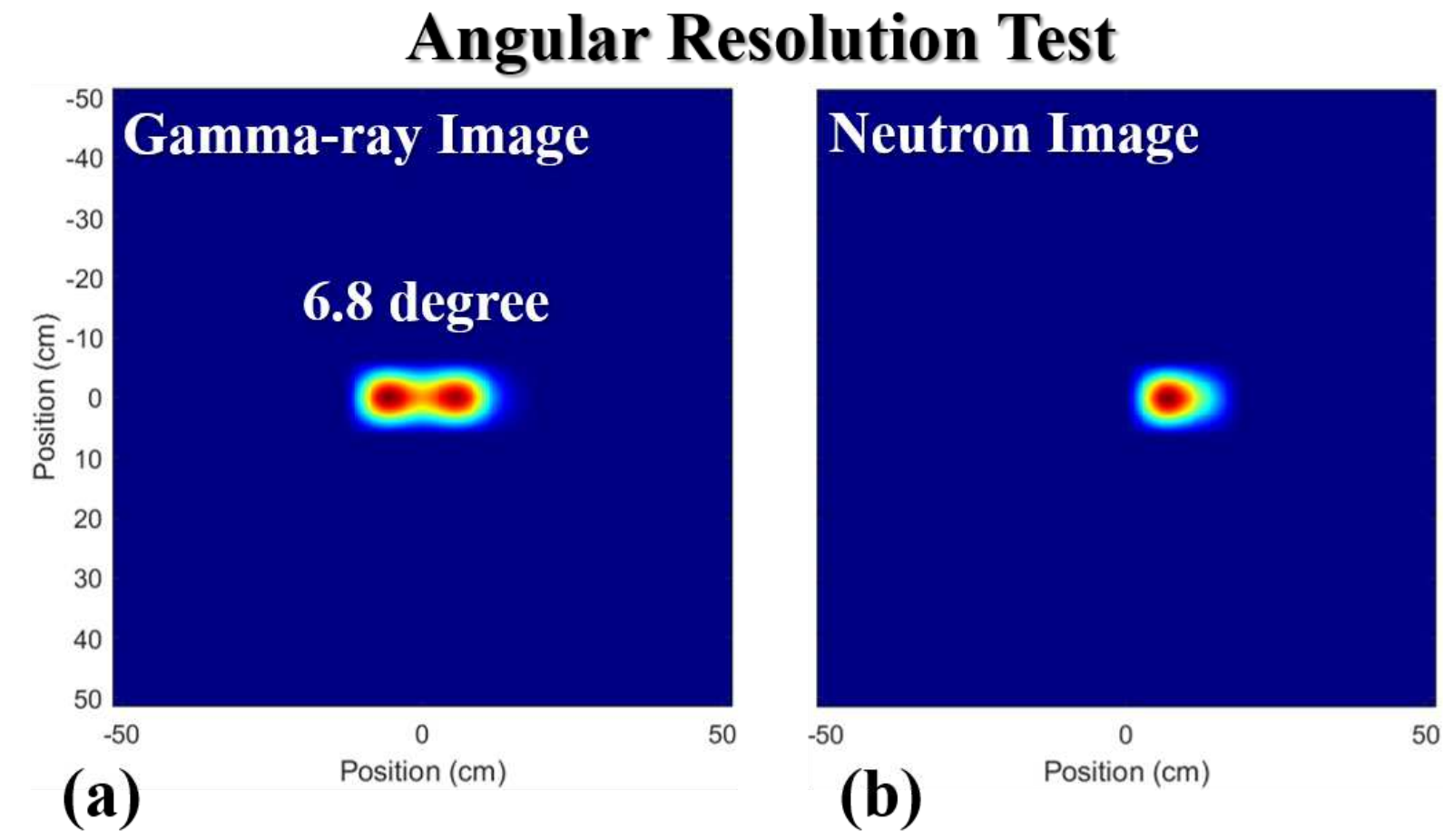

Figure 9. Angular separation test for the coded-aperture DPI system when exposed to a $3.5 \times$ $10^{5} \mathrm{n} / \mathrm{s}{ }^{252} \mathrm{Cf}$ spontaneous fission source at $75 \mathrm{~cm}$ distance and a ${ }^{137} \mathrm{Cs}$ source producing 30.16 $\mu \mathrm{R} / \mathrm{h}$ at $30 \mathrm{~cm}$ distance separated by $6.8^{\circ}$. Reconstructed images using gamma-ray events measured for $2 \min (a)$ and for neutron events for $4 \min (b)$ over the energy range above 50 keVee. 
Figure 10
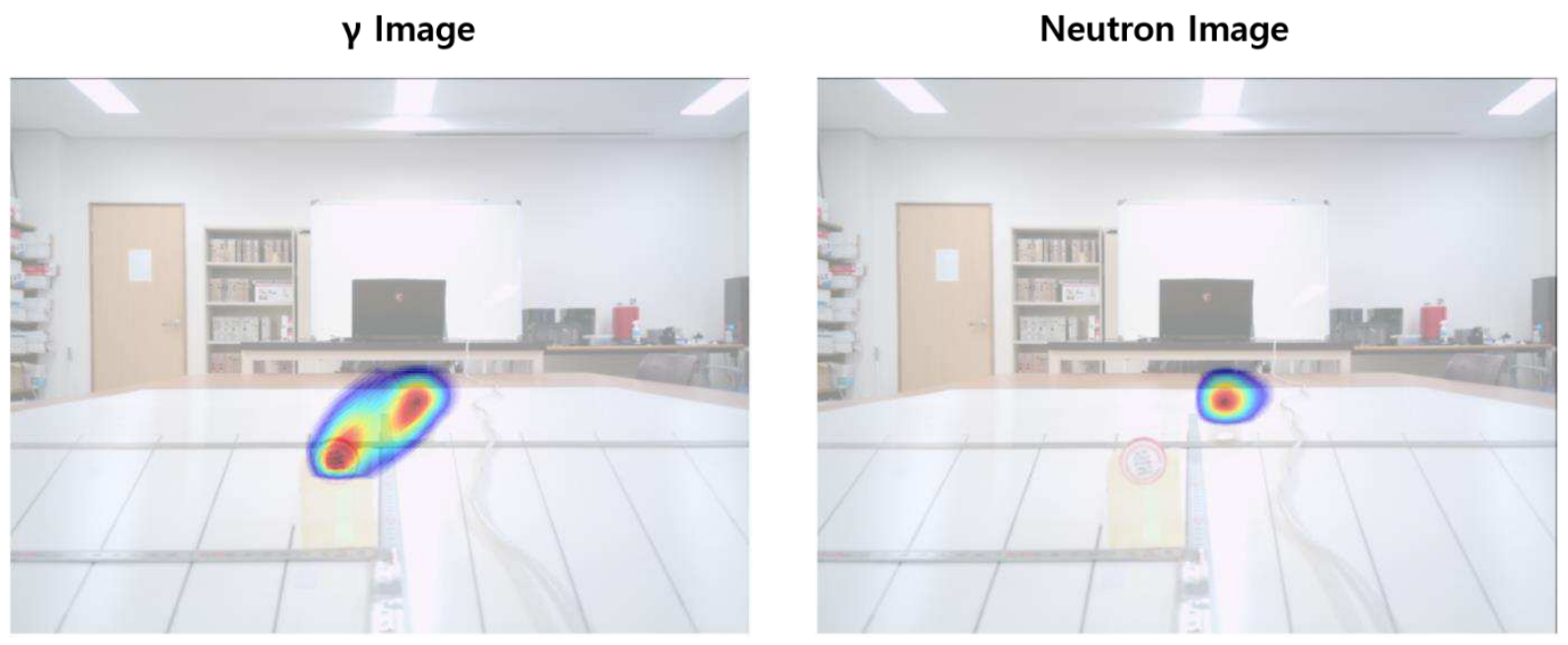

Figure 10. The optical camera images superimposed with the reconstructed images shown in Fig. 9.

Figure 11



(a)

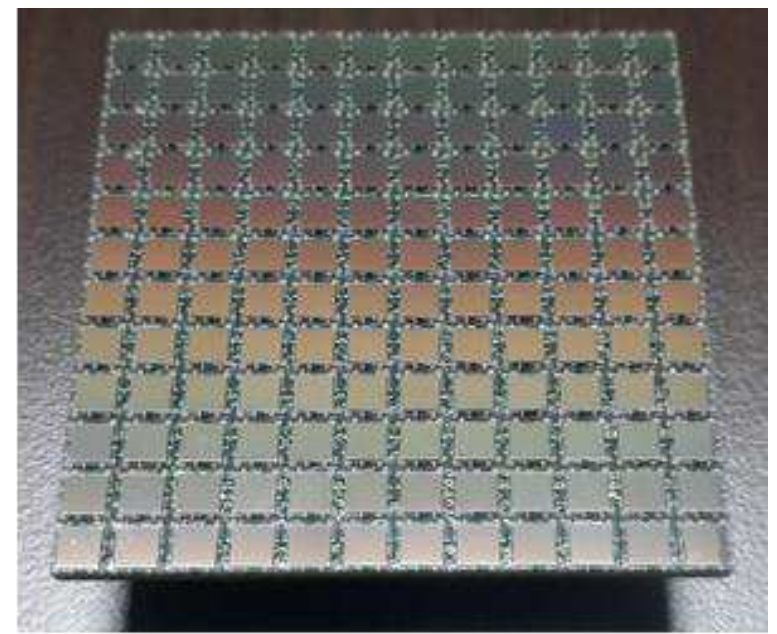

(b)

Figure 11. Stilbene scintillator array $\left(12 \times 12\right.$ pixels of $4 \times 4 \times 20 \mathrm{~mm}^{3}$ each $)(a)$, and SiPM array with the same number and area of pixels (b). 
Figure 12

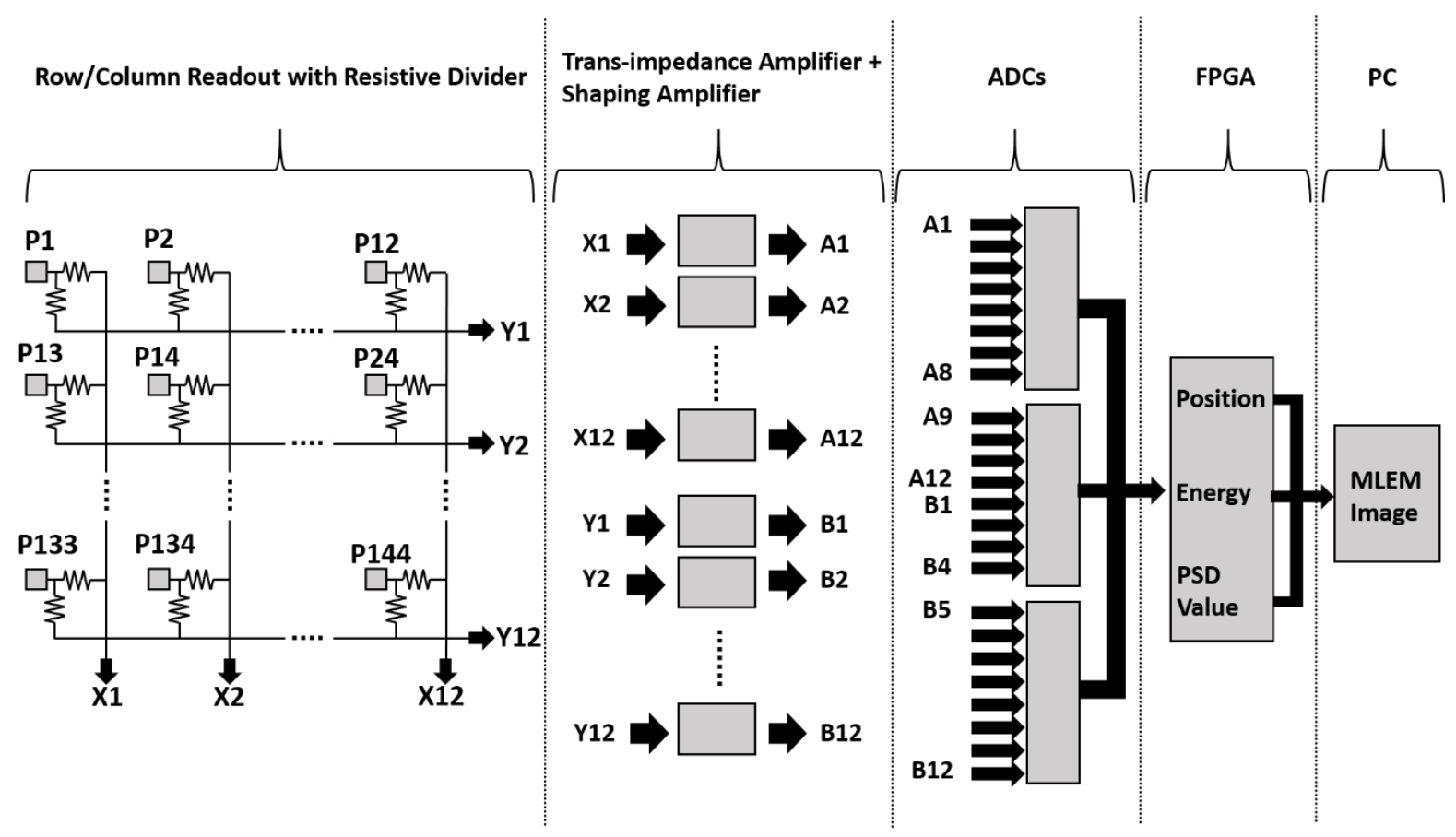

Figure 12. Hardware configuration with row/column readout and 3 ADCs for 144 pixels upon the SiPM array coupled to stilbene scintillator array implemented on coded-aperture hand-held imaging system. 
Figure 13



Figure 13. Prototype hardware configuration for driving a single sensor module. 
Figure 14

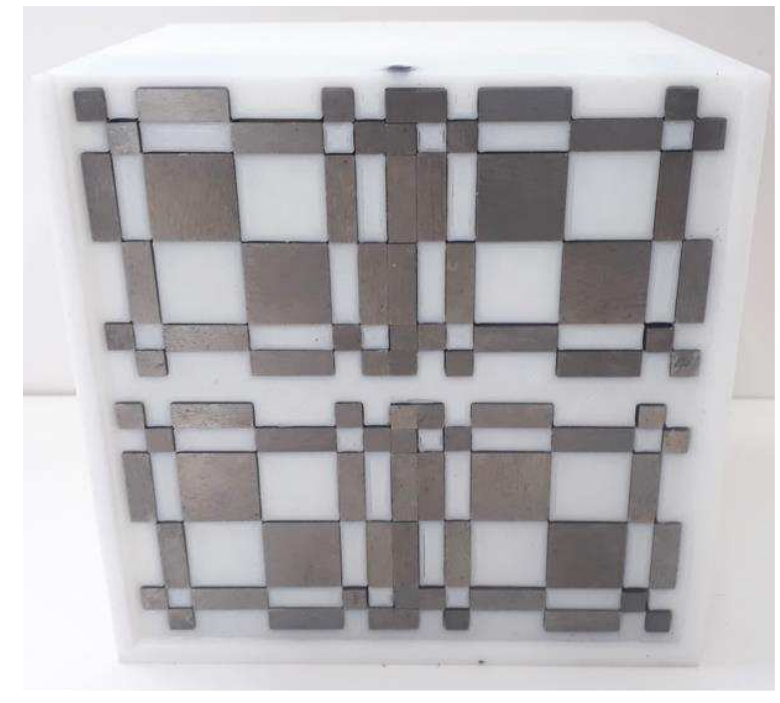

(a)

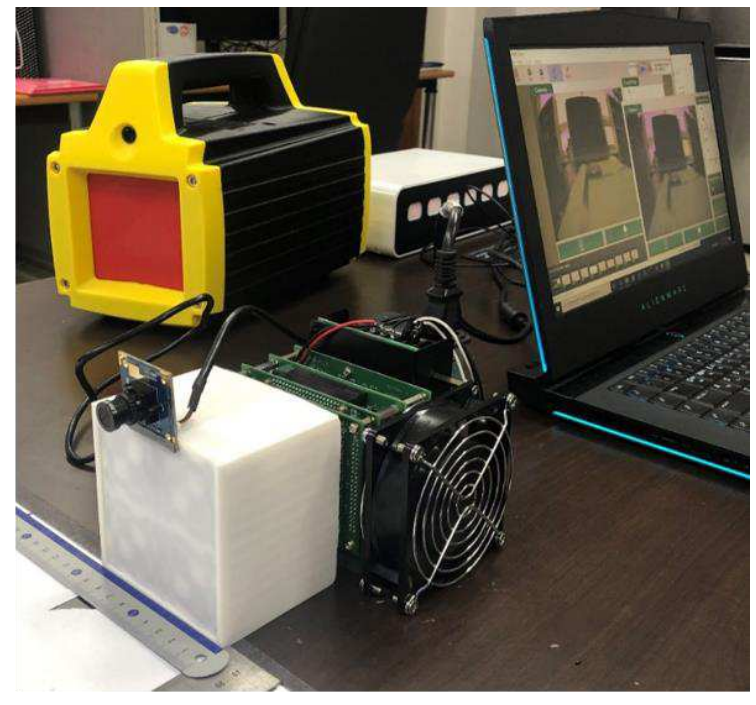

(b)

Figure 14. MURA mask (a), and developed hand-held DPI equipped with the MURA mask (b). 
Figure 15

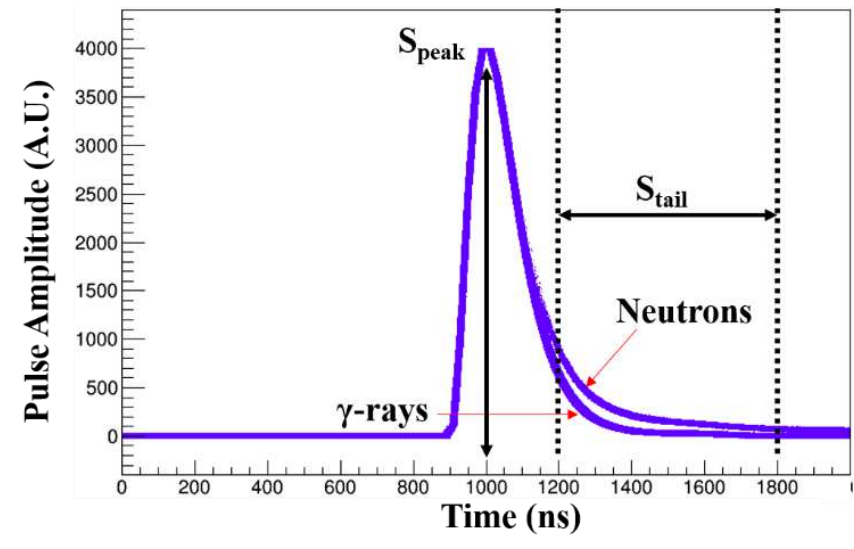

(a)



(b)

Figure 15. Illustration of the PSD method (a) and FOM evaluation (b) used in this study.

Table 1. Neutron image quality evaluated by using PSNR, NMSE, SSIM as increasing measurement time or the number of counts.

\begin{tabular}{cccc}
\hline $\begin{array}{c}\text { Number of neutron events } \\
\text { above 200 keVee }\end{array}$ & PSNR & NMSE & SSIM \\
\hline $330(1 \mathrm{~min})$ & 22.90 & $4.7 \times 10^{-3}$ & 0.81 \\
$1,000(3 \mathrm{~min})$ & 23.74 & $3.3 \times 10^{-3}$ & 0.85 \\
& 39.29 & $1.1 \times 10^{-4}$ & 0.99 \\
\hline
\end{tabular}


Tab1e 2. Gamma-ray image quality evaluated by using PSNR, NMSE, SSIM as increasing measurement time or the number of counts.

\begin{tabular}{cccc}
\hline $\begin{array}{c}\text { Number of gamma-ray events } \\
\text { above 200 keVee }\end{array}$ & PSNR & NMSE & SSIM \\
\hline $300(20 \mathrm{~s})$ & 25.17 & $1.6 \times 10^{-3}$ & 0.73 \\
$600(40 \mathrm{~s})$ & 26.82 & $1.3 \times 10^{-3}$ & 0.82 \\
& & & \\
$1,000(69 \mathrm{~s})$ & 50.65 & $8.6 \times 10^{-6}$ & 0.99 \\
\hline
\end{tabular}




\section{Figures}
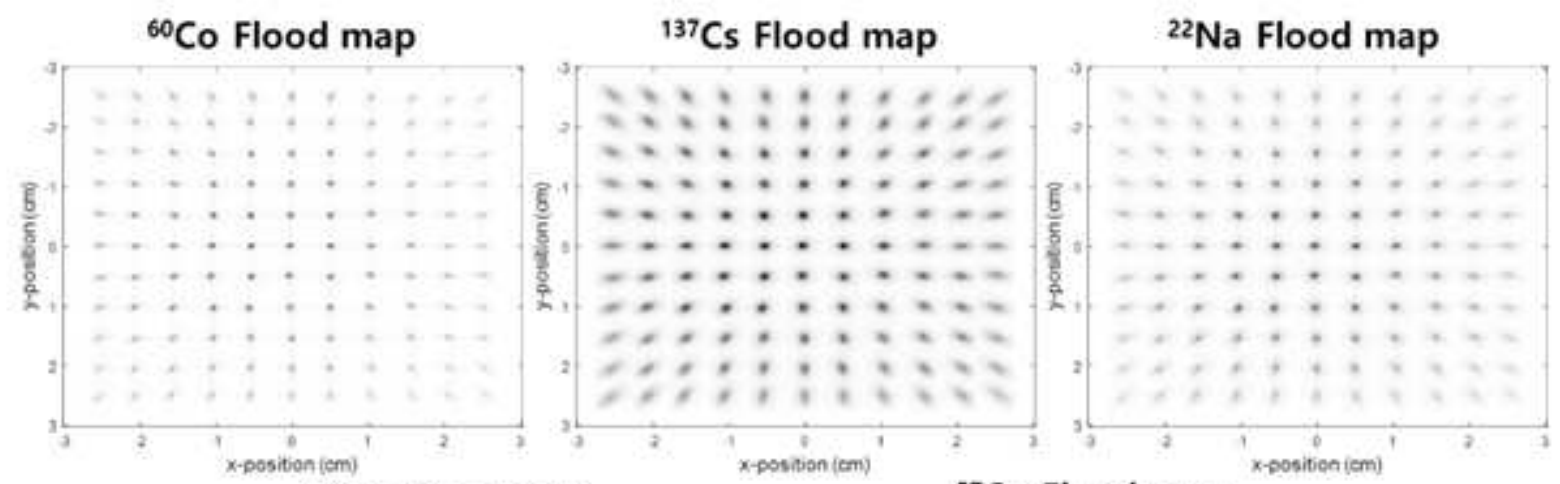

${ }^{133}$ Ba Flood map
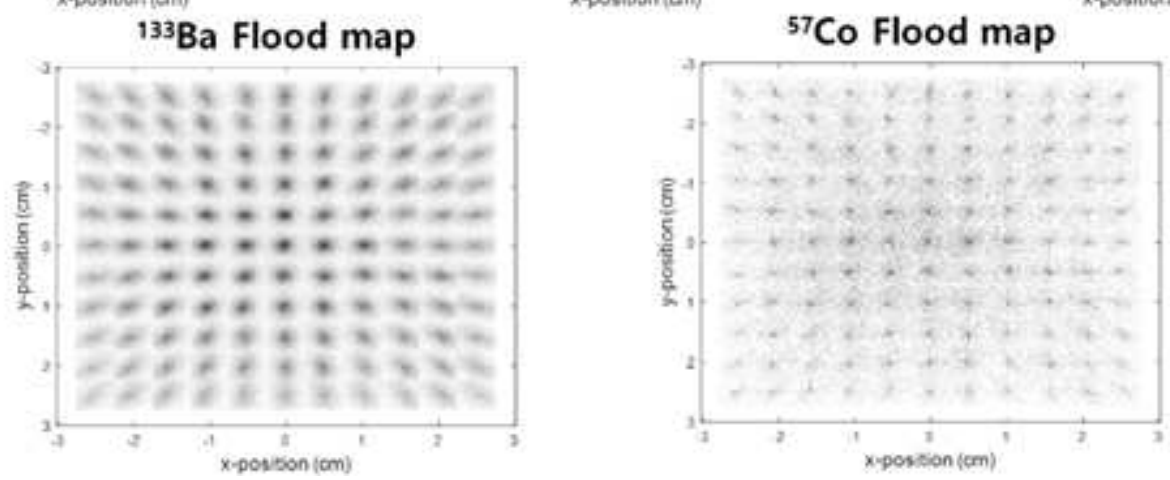

\section{Figure 1}

Measured 2D flood histograms of $12 \times 12$ pixels in stilbene-SiPM array module for $60 \mathrm{Co}, 137 \mathrm{Cs}, 22 \mathrm{Na}$, $133 \mathrm{Ba}$, and $57 \mathrm{Co}$ gamma irradiation. The source activity of each gamma-ray source was $0.312 \mathrm{MBq}$ and the $10 \mathrm{~cm}$ distant source was measured for 1 hour. The $12 \times 12$ stilbene scintillator had pixel dimensions $4 \times 4 \times 20 \mathrm{~mm} 3$.



(a)

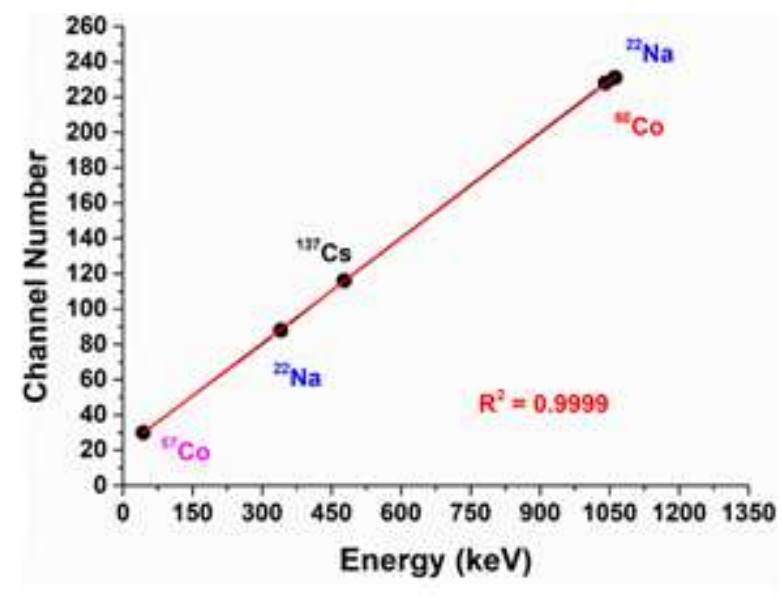

(b)

\section{Figure 2}

(a) Energy spectra of the stilbene scintillator array coupled to the SiPM array when irradiated by the gamma-ray sources shown. (b) Measured Compton edge height (in channel number) as a function of 
analytical Compton edge energy when the SiPM was operated at a $28 \mathrm{~V}$ bias voltage and a temperature of 28囚. The Compton edge height was calculated at $70 \%$ of the Compton peak for each of the gammaray sources.



Figure 3

PSD plot for 1,800,000 pulses with an energy threshold of 50 keVee produced by the stilbene-SiPM array module measuring a $3.5 \times 105 \mathrm{n} / \mathrm{s} 252 \mathrm{Cf}$ spontaneous fission source located $50 \mathrm{~cm}$ away from the front of detector. The dashed PSD cutoff line is established by the user. 


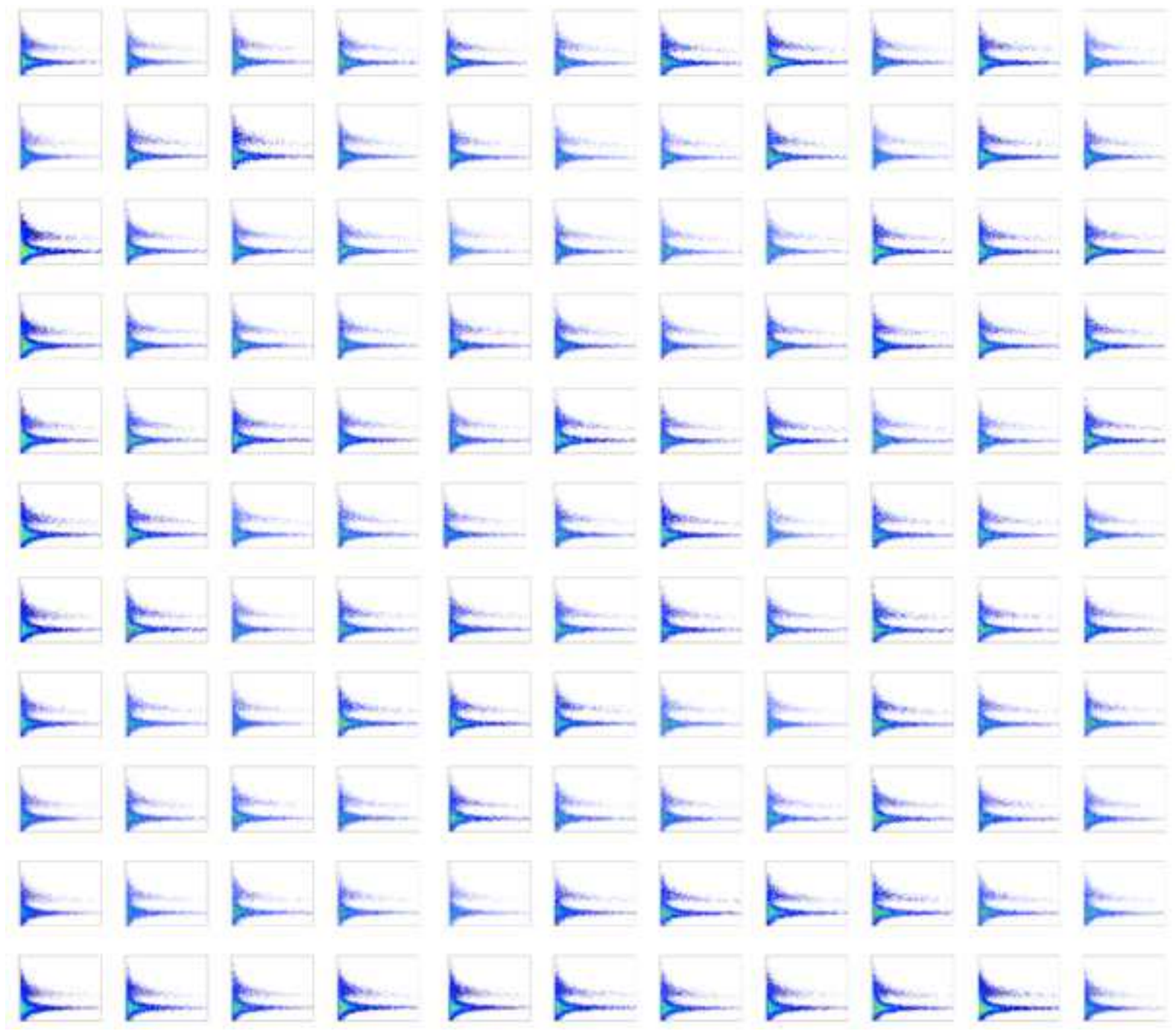

Figure 4

Pixel-by-pixel PSD plot for each $4 \times 4 \times 20 \mathrm{~mm} 3$ stilbene scintillator pixel from which the overall Fig. 3 distribution is generated. 



Figure 5

(a) The overall detector PSD plot for 1,500,000 pulses produced by the stilbene-SiPM module with a threshold of 50 keVee by measuring a $3.5 \times 105 \mathrm{n} / \mathrm{s} 252 \mathrm{Cf}$ spontaneous fission source at $75 \mathrm{~cm}$ distance and a 137Cs source producing $30.16 \mu \mathrm{R} / \mathrm{h}$ at $30 \mathrm{~cm}$ distance. (b) The distribution of PSD parameter at various energies ( $300 \pm 100$ keVee, $500 \pm 100 \mathrm{keVee}, 700 \pm 100 \mathrm{keVee}$ ) for the overall detector PSD plot. (c) The PSD plot that one of the pixels has and (d) the corresponding FOM values for the aforementioned three cases. 
(a)

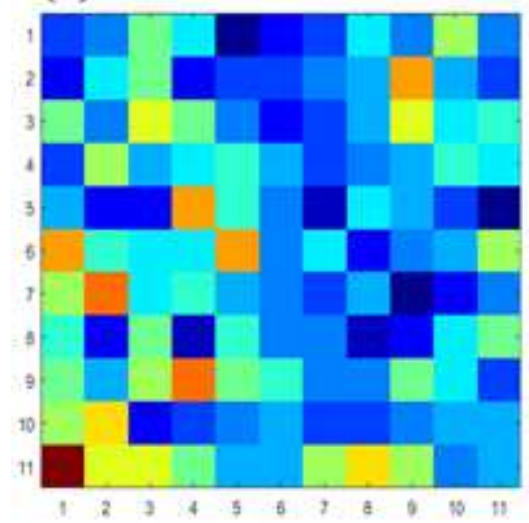

(d)

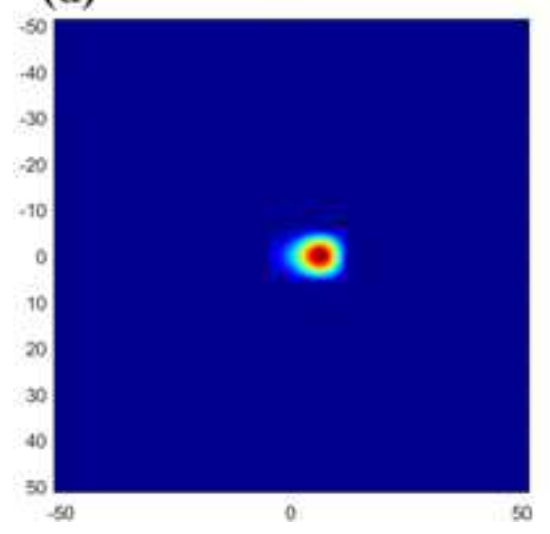

(b)

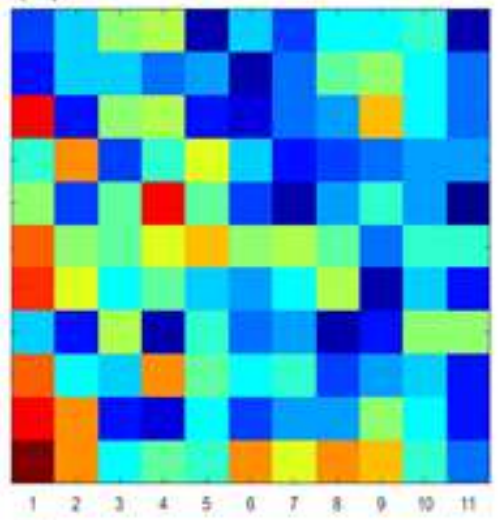

(e)

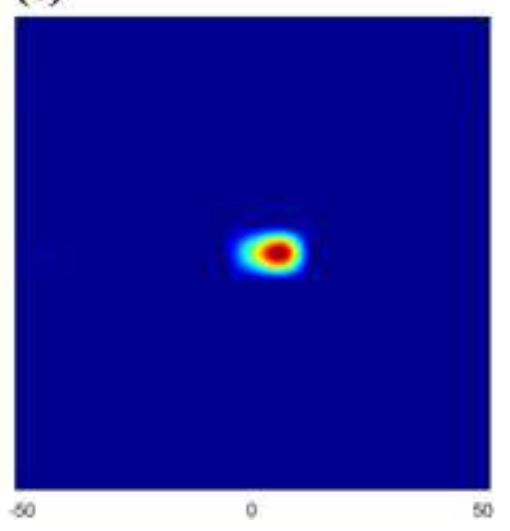

(c)

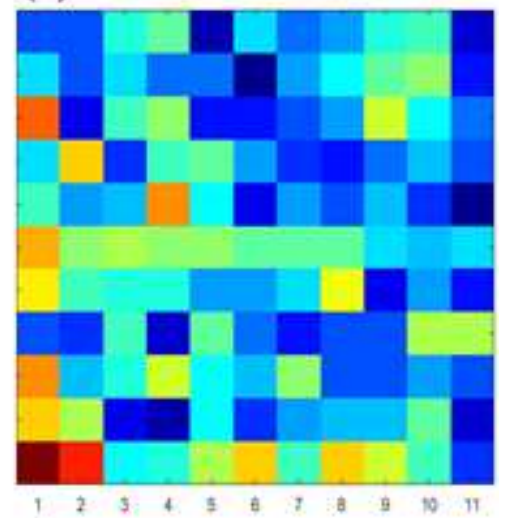

(f)

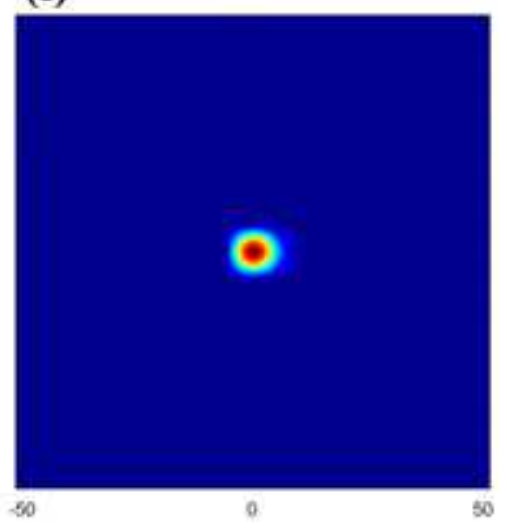

Figure 6

Comparison of reconstructed images of neutron when exposed to a $3.5 \times 105 \mathrm{n} / \mathrm{s} 252 \mathrm{Cf}$ spontaneous fission source at $100 \mathrm{~cm}$ distance. Detector map composes of the neutron events obtained for $1 \mathrm{~min}$ (330 events) (a), 3 min (1,000 events) (b), and 6 min (2,000 events) (c). The three detector maps correspond to the images reconstructed by using MLEM (d-f), respectively. 
(a)

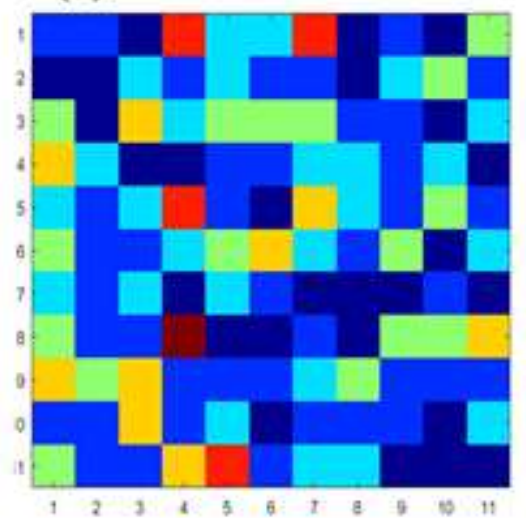

(d)

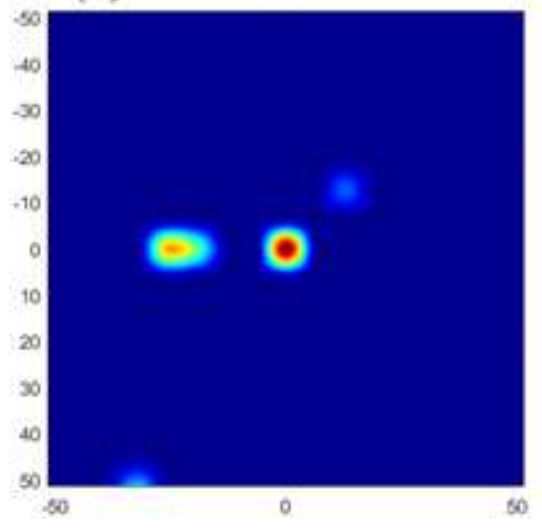

(b)

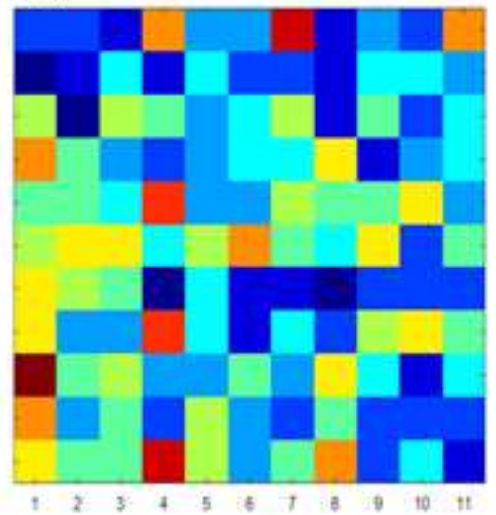

(e)

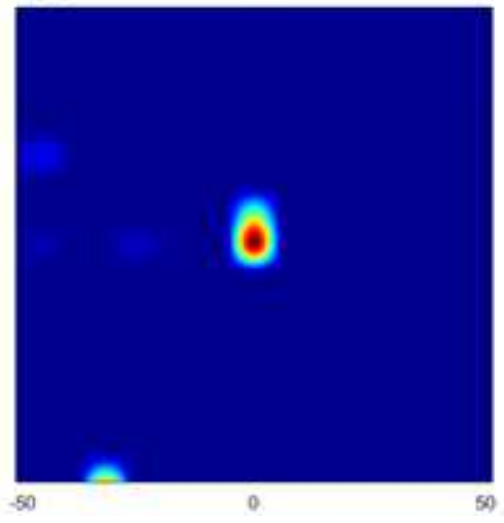

(c)

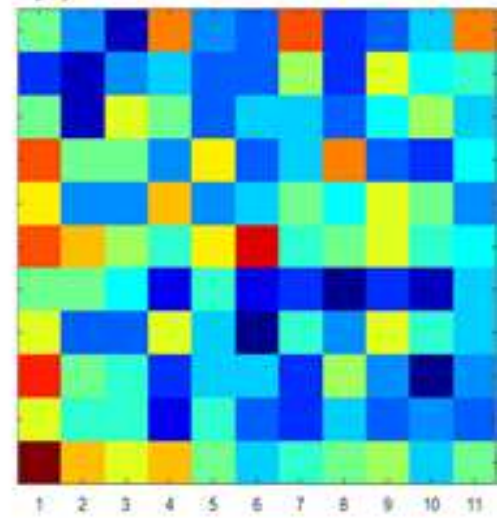

(f)

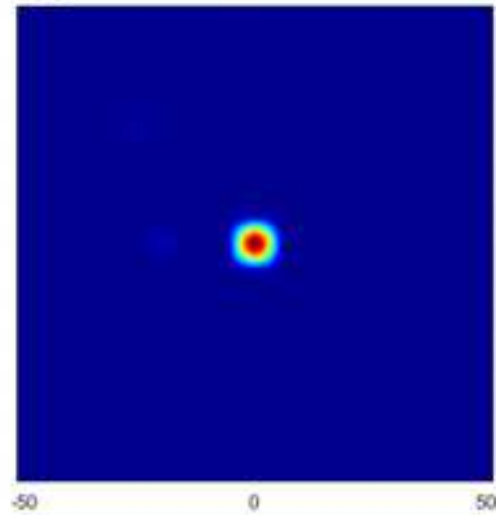

Figure 7

Comparison of reconstructed images of gamma-ray events when exposed to a 137Cs source producing $6.4 \mu \mathrm{R} / \mathrm{h}$ at $100 \mathrm{~cm}$ distance. The detector map composed of the gamma-ray events for (a) $20 \mathrm{sec}$ (300 events), (b) $40 \mathrm{sec}$ (600 events), and (c) $69 \mathrm{sec}$ (1,000 events). The three detector maps correspond to the images reconstructed by using MLEM (d-f), respectively. 


\section{Field of View Test}


Figure 8

Field of view (FOV) tests for the coded-aperture DPI system when a 137Cs source of $0.312 \mathrm{MBq}$ located at an angle of $-25^{\circ}(\mathrm{a})$, and at $+25^{\circ}(\mathrm{b})$ at $100 \mathrm{~cm}$ distance. Both reconstructed images are obtained using the gamma-ray events above the 50 keVee.

\section{Angular Resolution Test}

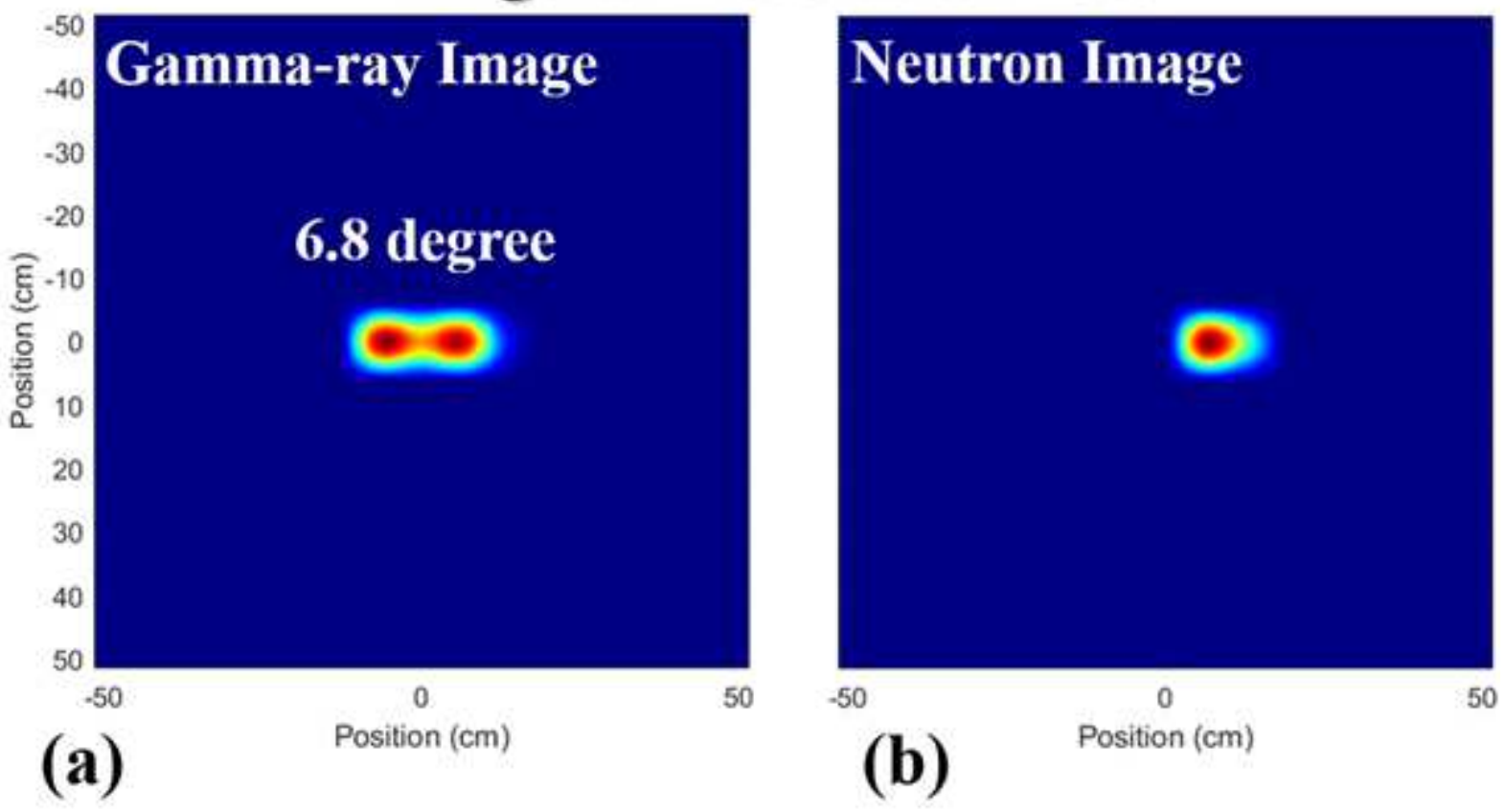

Figure 9 
Angular separation test for the coded-aperture DPI system when exposed to a $3.5 \times 105 \mathrm{n} / \mathrm{s} 252 \mathrm{Cf}$ spontaneous fission source at $75 \mathrm{~cm}$ distance and a 137Cs source producing $30.16 \mu \mathrm{R} / \mathrm{h}$ at $30 \mathrm{~cm}$ distance separated by $6.8^{\circ}$. Reconstructed images using gamma-ray events measured for 2 min (a) and for neutron events for 4 min (b) over the energy range above 50 keVee.



Neutron Image

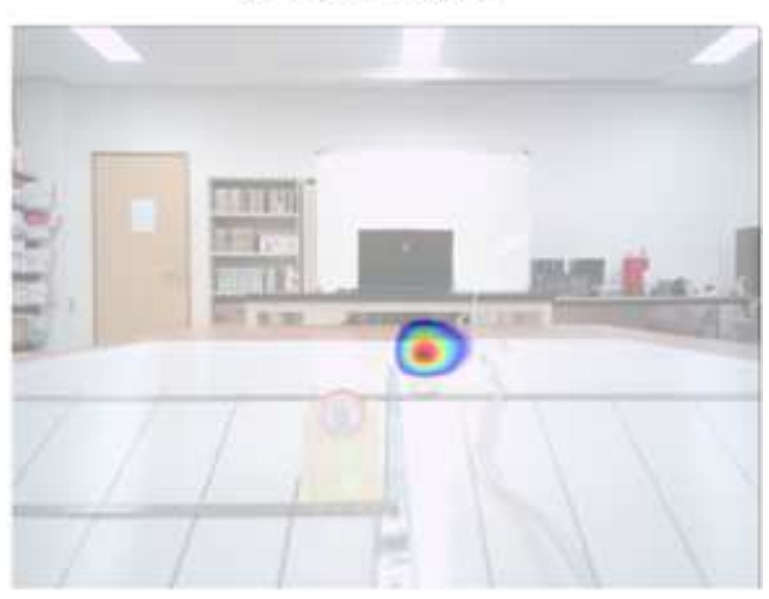

Figure 10

The optical camera images superimposed with the reconstructed images shown in Fig. 9.

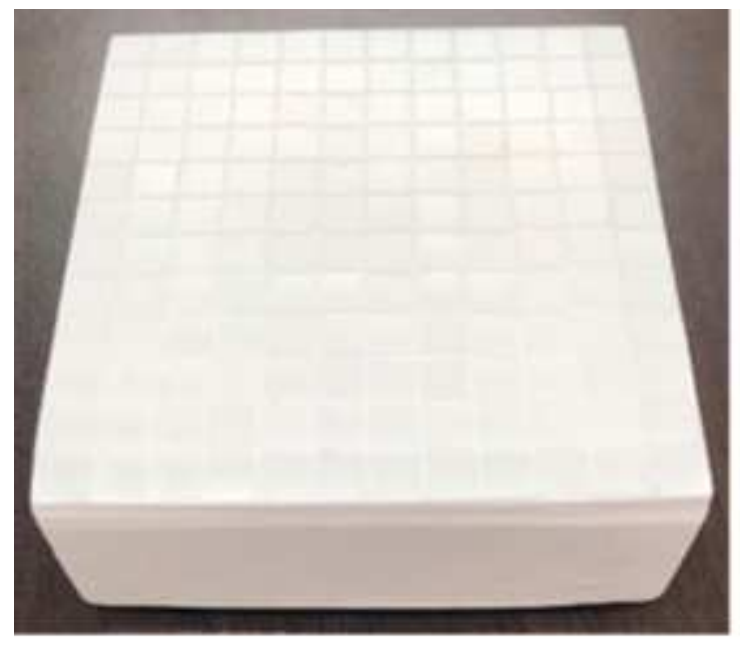

(a)

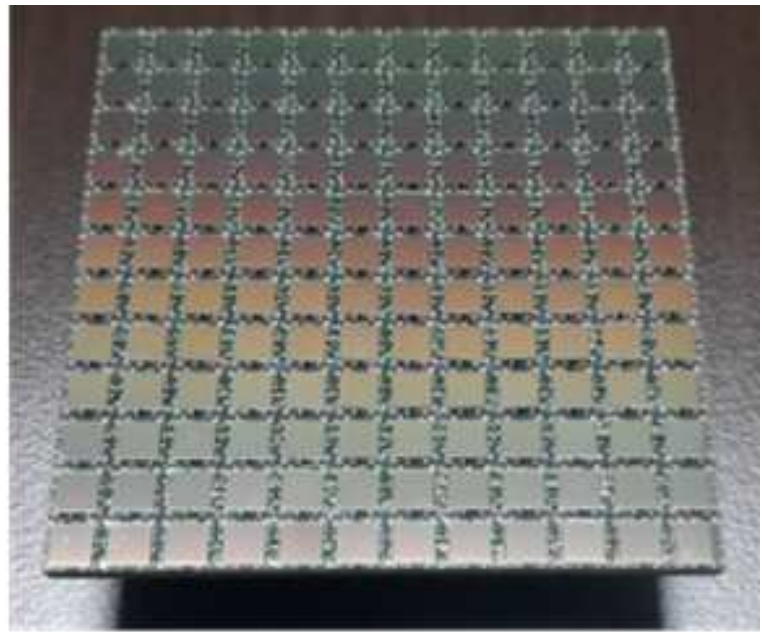

(b)

\section{Figure 11}

Stilbene scintillator array $(12 \times 12$ pixels of $4 \times 4 \times 20 \mathrm{~mm} 3$ each) (a), and SiPM array with the same number and area of pixels (b). 


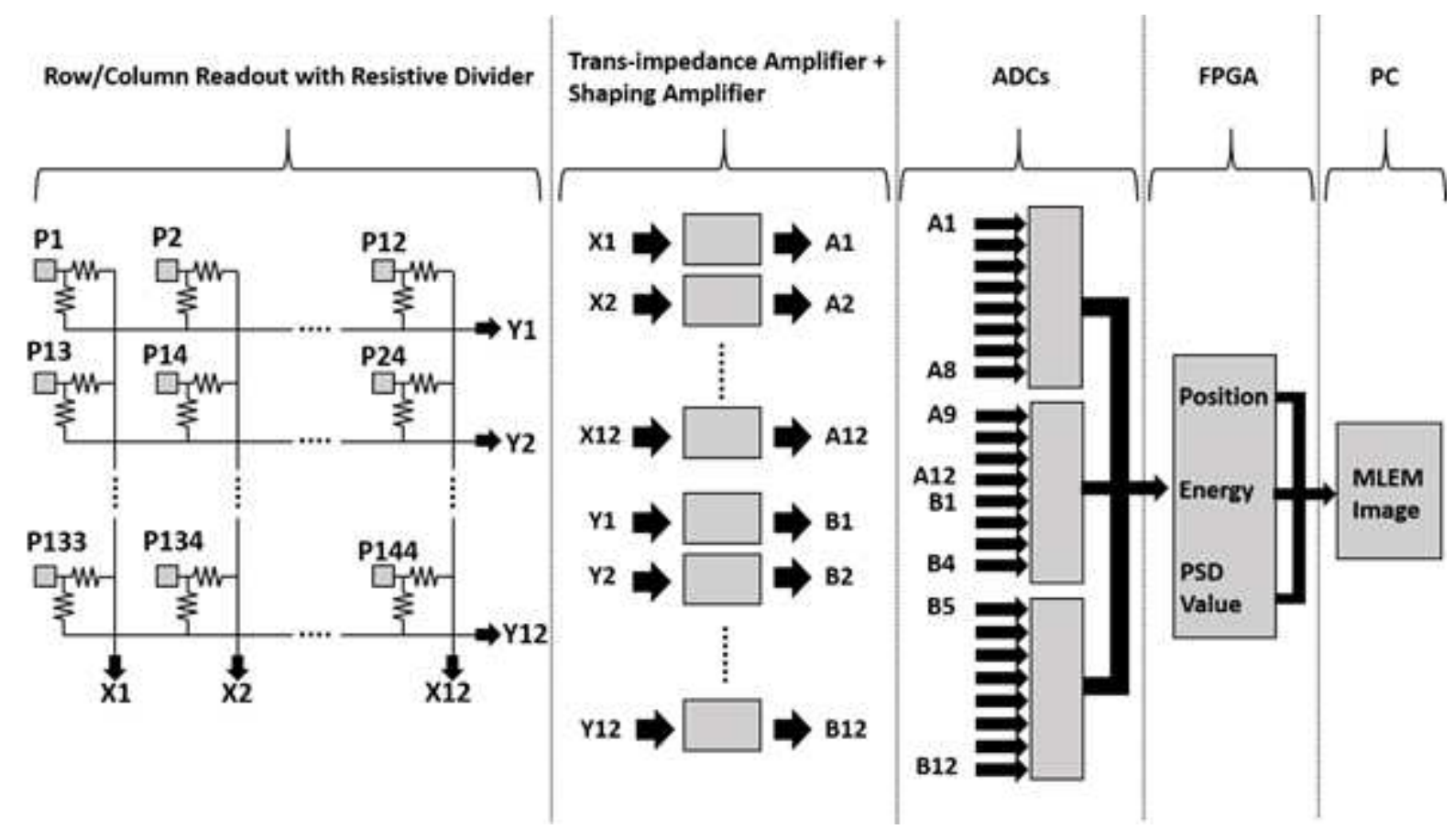

Figure 12

Hardware configuration with row/column readout and 3 ADCs for 144 pixels upon the SiPM array coupled to stilbene scintillator array implemented on coded-aperture hand-held imaging system.

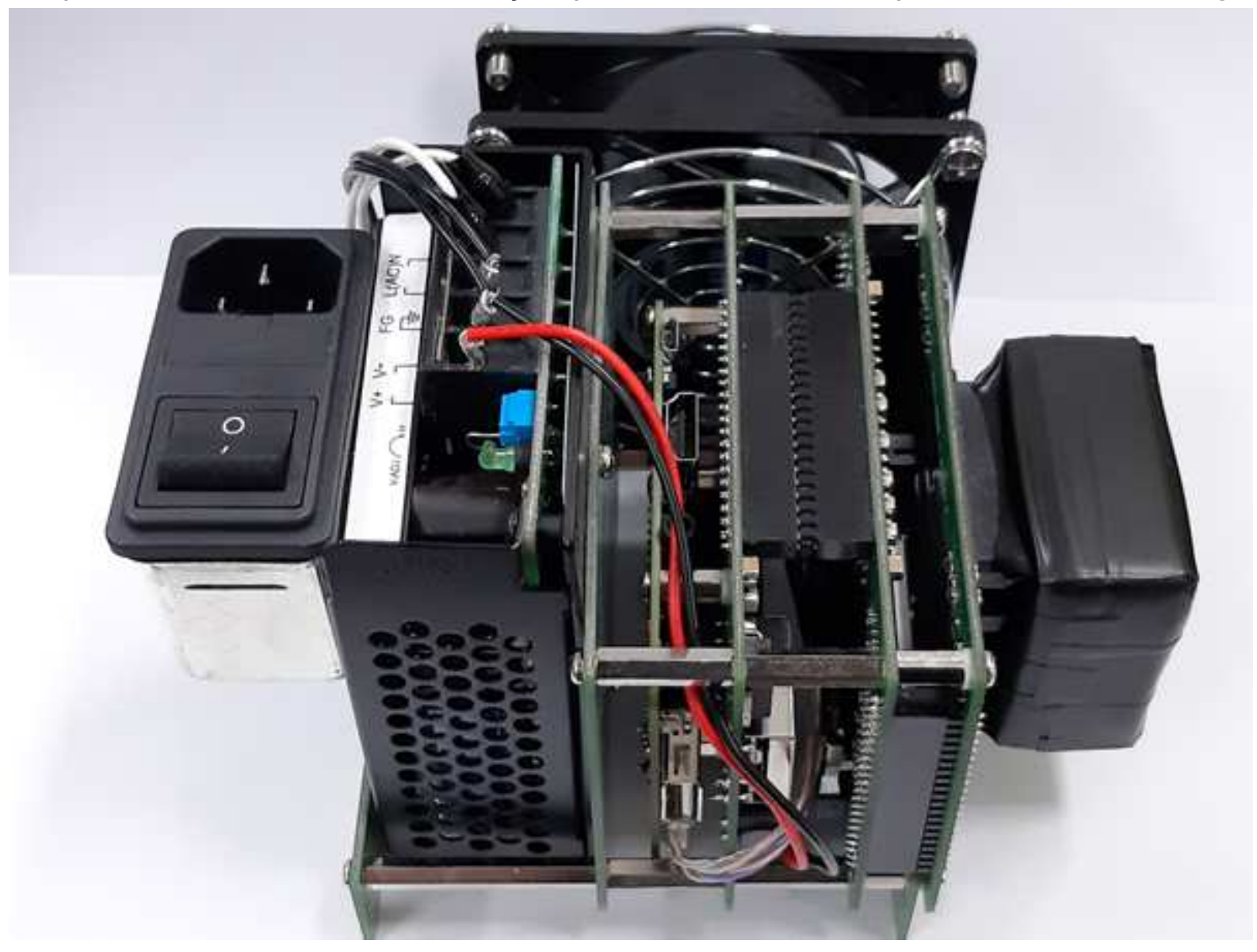

Figure 13 
Prototype hardware configuration for driving a single sensor module.



(a)

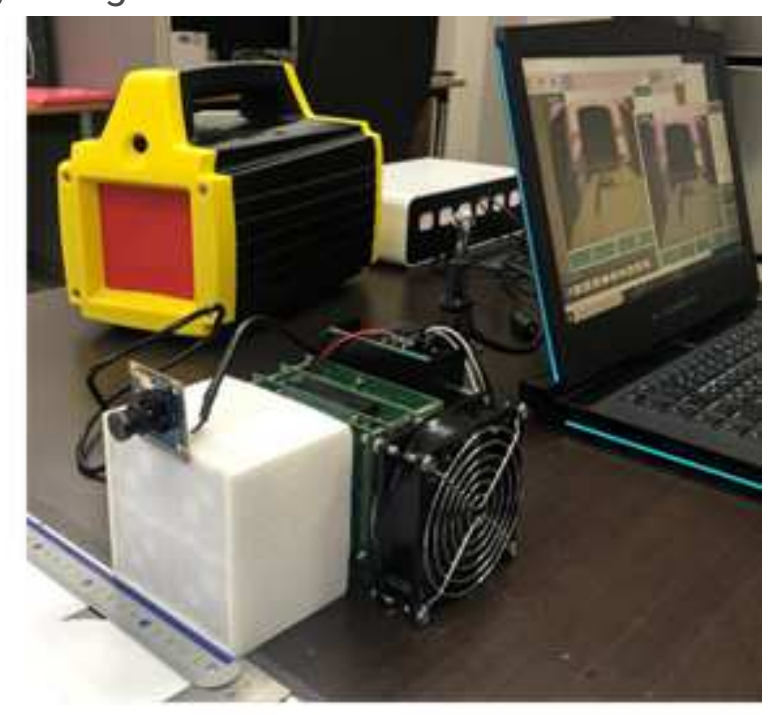

(b)

\section{Figure 14}

MURA mask (a), and developed hand-held DPI equipped with the MURA mask (b).

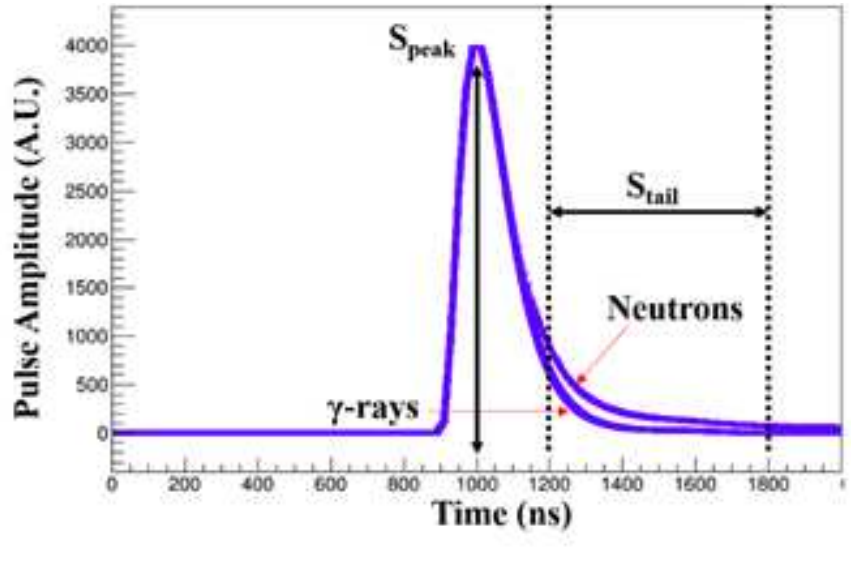

(a)

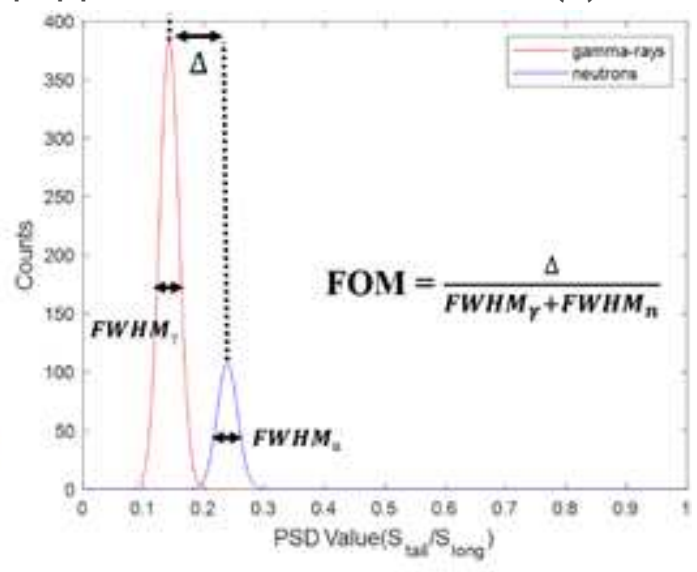

(b)

\section{Figure 15}

Illustration of the PSD method (a) and FOM evaluation (b) used in this study. 